\title{
Dynamic Inter-Operator Spectrum Sharing via Lyapunov Optimization
}

\author{
Satya Krishna Joshi, Student Member, IEEE, K. B. Shashika Manosha, Student Member, IEEE, \\ Marian Codreanu, Member, IEEE, and Matti Latva-aho, Senior Member, IEEE
}

\begin{abstract}
The problem of spectrum sharing between two operators in a dynamic network is considered. We allow both operators to share (a fraction of) their licensed spectrum band with each other by forming a common spectrum band. The objective is to maximize the gain in profits of both operators by sharing their licensed spectrum bands rather than using them exclusively, while considering the fairness among the operators. This is modeled as a two-person bargaining problem, and cast as a stochastic optimization. To solve this problem, we propose centralized and distributed dynamic control algorithms. At each time slot, the proposed algorithms perform the following tasks: 1) determine spectrum price for the operators; 2) make flow control decisions of users data; and 3) jointly allocate spectrum band to the operators and design transmit beamformers, which is known as resource allocation (RA). Since the RA problem is NP-hard, we have to rely on sequential convex programming to approximate its solution. To derive the distributed algorithm, we use alternating direction method of multipliers for solving the RA problem. Numerically, we show that the proposed distributed algorithm achieves almost the same performance as the centralized one. Furthermore, the results show that there is a trade-off between the achieved profits of the operators and the network congestion.
\end{abstract}

Index Terms - Co-primary spectrum sharing, dynamic control, network utility maximization, stochastic optimization, Lyapunov drift, bargaining problem, fairness, sequential convex programming, alternating direction method of multipliers (ADMM), distributed algorithm.

\section{INTRODUCTION}

$\mathbf{I}$ $\mathrm{N}$ THE wireless systems, the radio spectrum is divided into a set of disjoint blocks which are assigned (licensed) to different operators on an exclusive basis. The assignment of exclusive spectrum bands to operators gives each operator the right to control their spectrum bands. However, when the entire spectrum band is considered, the exclusive allocation strategy often leads to a low spectrum utilization, because the operators may have different spectrum demands over the time and some part of the spectrum band can be underutilized [1], [2]. Therefore, spectrum sharing between the operators is

This research was supported by the Finnish Funding Agency for Technology and Innovation (Tekes), Academy of Finland, Broadcom, Nokia, Huawei, Anite, Elektrobit, Nokia Foundation, and Tauno Tönning Foundation. The associate editor coor-dinating the review of this paper and approving it for publication was

P. Wang. (Corresponding author: Satya Krishna Joshi.)

The authors are with the Centre for Wireless Communications, University of Oulu, 90014 Oulu, Finland (e-mail: sjoshi@ee.oulu.fi; shkapuru@ee.oulu.fi; codreanu@ee.oulu.fi; matti.latva-aho@ee.oulu.fi). required for better spectrum utilization, and to cope with the rapidly increasing spectrum demand [3], [4].

The operators can share their spectrum band with each other in two basic ways [5]: orthogonal sharing and nonorthogonal sharing. In the orthogonal sharing, operators are allowed to operate in each others spectrum bands; but at any time instance one spectrum band can be used only by one operator. In contrast, in the non-orthogonal sharing, multiple operators are allowed to transmit on the same spectrum band at the same time and location. Here, the operators are required to coordinate their operation and choose transmission strategies to mitigate the inter-operator interference [6]. The performance of these two ways of spectrum sharing model depends on users' locations from base station (BS) [7].

Several orthogonal and non-orthogonal inter-operator spectrum sharing algorithms have been proposed in literature, e.g., [8]-[13]. Specifically, in [8] and [9] time division multiple access technique is considered, and the operators are allowed to lease their unused time slots with each other. A principle of last resort sharing is adopted in [8] and [9], i.e., an operator hires time slots only if its private portion is not sufficient to satisfy the QoS of its users. Moreover, the work in [9] considers sharing of infrastructure (BSs) among the operators. The use of a common spectrum pool [14], [15] to share the spectrum among operators is investigated in [10], [11], and [13]. Two operators are considered in [10] and [11] and distributed algorithms using the non-cooperative game theory [16] are proposed. In general, the non-cooperative game theoretic approach leads to a stable operating point, and it is known as Nash equilibrium (NE). However, in the context of spectrum sharing the NE point is often seen as an inefficient operating point, as the operators' performance can be further improved over it [12], [17], [18]. In [13] the co-primary shared access model [3] is adopted, and several heuristic centralized and distributed algorithms are proposed. All works in [8]-[13] consider single antennal transmitters and receivers.

The problem of spectrum sharing among operators in multiple-input single-output antenna wireless systems have been considered in [19]-[22]. Specifically, in [19] a distributed algorithm is proposed using cooperative game theory [16]. In [20] the problem is studied by using both cooperative and non-cooperative (competitive) game theoretic approach, and a significant gain by operators cooperation has been demonstrated. For cooperative spectrum sharing, various transmit beamforming techniques to manage the inter-operator interference have been proposed in [21] and [22]. 
To the best of our knowledge all existing spectrum sharing algorithms consider a static case (i.e., the spectrum sharing problem for a given instance). Thus, when these algorithms are applied over a period of time to a dynamic network, they may yield suboptimal performance and also may not ensure the stability of the network [23, Sec. 4.1].

In this paper the problem of dynamic spectrum sharing between two wireless cellular network operators is considered. We assume a co-primary setup [3], [24], where operators share a fraction of their licensed spectrum by forming a common spectrum pool, which is dynamically allocated to the operators according to their channel qualities, traffic demands, past activities, etc.

Clearly, by sharing the spectrum instead of using it individually the operators can increase their own profits, but this gives rise to a new problem: how to distribute the surplus (i.e., the increase in the profit) fairly among the operators, so that they have incentive to share their licensed spectrum with each other. For example, the total (or sum) profit can be maximized by allocating each portion of the shared spectrum to the operator that can make the most profit out of it. However, such a simplistic strategy does not make much sense since it may lead to a highly unfair outcome, where some operators may even diminish their profit instead of increasing it.

This problem of fair surplus management has been intensively studied, especially in economics, and a solution has been proposed by Nash in 50's [25]. The bargaining problem proposed in [25] has shown that under a reasonable set of axioms, a fair operating point can be obtained by maximizing the product of the incremental profit gains, with respect to the case when the agents do not cooperate. We adopt this definition of fairness to share the licensed spectrum band between two cellular network operators.

Our goal is to propose a dynamic network control mechanism (algorithm) which decides at each time slot: 1) portion of spectrum band for each operator from the common spectrum pool; 2) beamformers, powers, transmission rates, admitted data, etc., for users; and 3) possible inter-operators payments, such that the surplus obtained from cooperation is fairly shared among the operators. To do this, we formulate (or cast) the spectrum sharing problem as a Nash bargaining game [17], [25]-[28], and then we use the Lyapunov drift plus penalty framework [23], [29] to derive a dynamic control algorithm.

The main contribution of the paper is to propose both centralized and distributed dynamic control algorithms to share the common spectrum pool between two operators. Our proposed dynamic algorithms perform at each time slot the following tasks: 1) determine the spectrum price for the operators; 2) make flow control decisions for the users data; and 3) jointly allocate spectrum band to the operators and design the transmit beamformers and powers, which is known as resource allocation (RA). The RA problem leads to a general weighted sum-rate maximization problem over the instantaneous achievable rate region [23], [29], and thus it is NP-hard [30]. Since the RA problem is NP-hard, in the centralized algorithm we use the sequential convex programming (SCP) [31] to approximate the solution of the RA problem. To derive the distributed algorithm, we use the alternating direction method of multipliers (ADMM) [32] in conjunction with the SCP for solving the RA problem. Numerically, we show that the proposed distributed dynamic control algorithm achieves almost the same performance as the centralized one. Furthermore, the results show that there is a trade-off between the achieved profits of the operators and the network congestion.

The remainder of this paper is organized as follows. In Section II, the considered system model and problem formulation are described. We present the steps of the proposed dynamic control algorithms in Section III. Centralized and distributed algorithms to solve the RA problem are presented in Section IV and Section V, respectively. The numerical results are presented in Section VI, and Section VII concludes our paper.

Notations: All boldface lower case and upper case letters represent vectors and matrices, respectively, and calligraphy letters represent sets. The notation $\mathbb{C}^{n}$ denotes the set of complex $n$-vectors, $|x|$ denotes the absolute value of the scalar $x,|x|$ denotes the cardinality of set $x,\|\mathbf{x}\|_{2}$ denote the Euclidean norm of the vector $\mathbf{x}$, I denotes the identity matrix, $(x)^{+}$denotes max $(x, 0), \emptyset$ denotes the empty set, and $\mathcal{C N}(\mathbf{m}, \mathbf{C})$ denotes the complex circular symmetric Gaussian vector distribution with mean $\mathbf{m}$ and covariance matrix $\mathbf{C}$. For symmetric matrix $\mathbf{X}, \mathbf{X} \succeq 0$ signifies that $\mathbf{X}$ is positive semidefinite. The superscripts $(\cdot)^{\mathrm{H}}$ and $(\cdot)^{\mathrm{T}}$ are used to denote the Hermitian transpose and the transpose of a matrix, and $(\cdot)^{\star}$ is used to denote a solution of an optimization problem.

\section{System Model AND Problem Formulation}

We consider a downlink wireless network consisting of a cell with two coexisting BSs, belonging to two different operators. The set of BSs is denoted by $\mathcal{N}$, and we label them with the integer values $n=1,2$. Each $\mathrm{BS}$ is equipped with $T$ transmit antennas, and each user is equipped with single receive antenna. We denote the set of all users in $n$th BS by $\mathcal{L}(n)$, and we label them with the integer values $l=1, \ldots, L_{n}$. Let each operator share equal ${ }^{1}$ amount of spectrum band $B \mathrm{~Hz}$ with the other operator. Hence, a total spectrum of bandwidth $2 B \mathrm{~Hz}$ is available for both operators. Furthermore, we assume that the total spectrum band $2 B \mathrm{~Hz}$ is split into $S$ subchannels. The set of subchannels is denoted by $\mathcal{S}$, and we label them with the integer values $s=1, \ldots, S$. Let the bandwidth of $s$ th subchannel be $w_{s} \mathrm{~Hz}$, and we assume that it is smaller than a coherence bandwidth. See Fig. 1 for a functional architecture of the considered co-primary spectrum sharing setup.

The network is assumed to be operating in slotted time with slots normalized to integer values $t \in\{1,2, \ldots\}$. At each time slot, a network controller partitions the $S$ subchannels orthogonally between the operators (i.e., between the two BSs). ${ }^{2}$ Let the set of subchannels allocated to $n$th BS during time slot $t$ be $S(n, t)$, and we label them with the integer values $s=1, \ldots, S_{n}(t)$.

Let $p_{n l, s}(t)$ and $\mathbf{v}_{n l, s}(t)$ denote the power and direction of the transmit beamformer associated with $l$ th user of BS $n$

\footnotetext{
${ }^{1}$ The work can be easily generalized to the case where operators share different portions of the spectrum bands with each other.

${ }^{2} \mathrm{We}$ use the terminologies BS and operator interchangeably.
} 


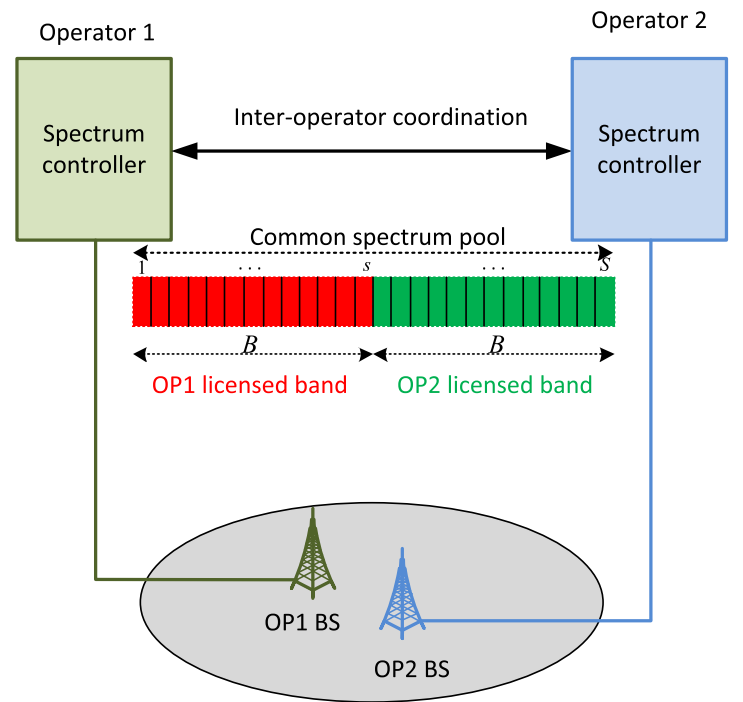

Fig. 1. High level functional architecture for co-primary spectrum sharing [3], [24], [33]: Two operators (OP) share a common spectrum pool in a same geographical area. Usage conditions of common spectrum pool is defined with a mutual agreement between operators. Spectrum controller is responsible for inter-operator spectrum coordination. We refer the interested reader to [24] for more detail on co-primary sharing architecture for a denser network.

in subchannel $s$ during time slot $t$. We assume $\mathbf{v}_{n l, s}(t)$ is normalized such that $\left\|\mathbf{v}_{n l, s}(t)\right\|_{2}=1$ for all $n \in \mathcal{N}, l \in \mathcal{L}(n)$, and $s \in \mathcal{S}$. Furthermore, let $\mathbf{m}_{n l, s}(t)=\sqrt{p_{n l, s}(t)} \mathbf{v}_{n l, s}(t)$, then the signal-to-interference-plus-noise ratio of $l$ th user of BS $n$ in subchannel $s$ during time slot $t$ is given by

$\Gamma_{n l, s}\left(\mathbf{m}_{n}(t)\right)=\frac{\left|\mathbf{h}_{n l, s}^{\mathrm{H}}(t) \mathbf{m}_{n l, s}(t)\right|^{2}}{N_{0} w_{s}+\sum_{j \in \mathcal{L}(n), j \neq l}\left|\mathbf{h}_{n l, s}^{\mathrm{H}}(t) \mathbf{m}_{n j, s}(t)\right|^{2}}$,

where $\mathbf{h}_{n l, s}^{\mathrm{H}}(t) \in \mathbb{C}^{1 \times T}$ is the channel vector from $n$th BS to its $l$ th user in subchannel $s, N_{0}$ is a noise power spectral density, and the notation $\mathbf{m}_{n}(t)$ denotes a vector obtained by stacking $\mathbf{m}_{n l, s}(t)$ for all $l \in \mathcal{L}(n)$ and $s \in \mathcal{S}(n, t)$ on top of each other, i.e., $\mathbf{m}_{n}(t)=\left[\mathbf{m}_{n 1,1}(t)^{\mathrm{T}}, \ldots, \mathbf{m}_{n L_{n}, S_{n}(t)}(t)^{\mathrm{T}}\right]^{\mathrm{T}}$.

In this paper, we consider the case where all receivers are using single-user detection (i.e., a receiver decodes its intended signal by treating all other interfering signals as noise), and assume that the achievable rate of $l$ th user of $n$th BS during time slot $t$ is given by [34, Ch. 5]

$$
\begin{aligned}
& r_{n l}(t) \\
& \triangleq r_{n l}\left(S(n, t), \mathbf{m}_{n}(t)\right) \\
& =\sum_{s \in \mathcal{S}(n, t)} w_{s} \log _{2}\left(1+\frac{\left|\mathbf{h}_{n l, s}^{\mathrm{H}}(t) \mathbf{m}_{n l, s}(t)\right|^{2}}{N_{0} w_{s}+\sum_{j \in L(n), j \neq l}\left|\mathbf{h}_{n l, s}^{\mathrm{H}}(t) \mathbf{m}_{n j, s}(t)\right|^{2}}\right) .
\end{aligned}
$$

Furthermore, we assume that the power allocation is subject to a maximum power constraint $\sum_{l \in \mathcal{L}(n)} \sum_{s \in S(n, t)}\left\|\mathbf{m}_{n l, s}(t)\right\|_{2}^{2} \leq$ $p_{n}^{\max }$ for each BS $n \in \mathcal{N}$.

\section{A. Spectrum Pricing}

At each time slot, the common spectrum pool $2 B \mathrm{~Hz}$ (i.e., the set of $S$ subchannels) is partitioned between the operators. The total spectrum band allocated to operator $n \in \mathcal{N}$ during time slot $t$ is $\sum_{s \in S(n, t)} w_{s}$. We assume that both operators can use up to the amount of spectrum that they put in the spectrum pool without any payment. But, the operator pays for an extra band of spectrum, if it uses more spectrum than it has put in the common spectrum pool, to the other operator. Specifically, if spectrum band used by $n$th operator $\sum_{s \in S(n, t)} w_{s}$ is more than $B \mathrm{~Hz}$, operator $n$ pays to the other operator (i.e., opponent of $n$th operator) for the extra band of spectrum $\left(\sum_{s \in \mathcal{S}(n, t)} w_{s}-B\right) \mathrm{Hz}$. The amount to be paid is determined by the pricing rule established by the operators.

Let $q_{n}(t)$ be the per-unit price of spectrum during time slot $t$ to charge the $n$th operator's opponent for using the extra spectrum band. To simplify the notation, let us use $\underline{n}$ to denote the opponent of $n$th operator. ${ }^{3}$ Then the payment from operator $n \in \mathcal{N}$, for using the extra band of spectrum, to its opponent is $q_{\underline{n}}(t)\left(\sum_{s \in S(n, t)} w_{s}-B\right)^{+}$.

With this spectrum pricing rule, operators with both low and high spectrum demands can be benefitted. For example, an operator with a high spectrum demand can have access to more spectrum than it owns; while an operator with a low spectrum demand (or, no spectrum demand at all) is getting paid for leasing its spectrum. Even in the case when both operators use the same amount of spectrum that they have put to the common spectrum pool (i.e., in the case of equal spectrum demand), they can still gain as the operators have an opportunity to access subchannels with better channel quality from the common spectrum pool, free of charge.

\section{B. Network Queuing and Time Average Profit}

We consider a network utility maximization framework similar to the one considered in [23, Sec. 5.1], [29, Ch. 5]. Specifically, exogenously arriving data is not immediately admitted to the network layer of the BSs. Instead, the exogenous data is first placed in the transport layer storage reservoirs. Let $D_{n l}(t)$ represent the transport layer storage backlog of $l$ th user of BS $n$ during time slot $t$, and $\lambda_{n l}(t)$ represents the amount of data that exogenously arrives to it. Then, at each time slot a flow control decision is made, and the amount of each user data to be admitted to the network layer from reservoir $D_{n l}(t)$ is decided. Let $a_{n l}(t)$ denote the amount of data of $l$ th user of $n$th BS that is admitted to the network layer from the reservoir $D_{n l}(t)$ during time slot $t$.

We assume that only the data currently available in $D_{n l}(t)$ at the beginning of slot $t$ can be admitted to the network layer during that slot. The transport layer storage reservoir may not always have data to be admitted to the network layer, and hence the flow rate $a_{n l}(t)$ is subject to a constraint $a_{n l}(t) \leq D_{n l}(t)$ for each user. Then the dynamics of the transport layer storage reservoir $D_{n l}(t)$ from one time slot to the next can be expressed as ${ }^{4}$

$$
D_{n l}(t+1)=\max \left[D_{n l}(t)-a_{n l}(t), 0\right]+\lambda_{n l}(t),
$$

for all $n \in \mathcal{N}$ and $l \in \mathcal{L}(n)$. Here, we assume that the exogenously arriving data $\left\{\lambda_{n l}(t)\right\}_{n \in \mathcal{F}, l \in \mathcal{L}(n)}$ can have arbitrary

\footnotetext{
${ }^{3}$ For operator $n=1$, its opponent is $\underline{n}=2$. Similarly, for operator $n=2$, its opponent is $\underline{n}=1$.

${ }^{4}$ In the case transport layer storage reservoir have a finite size $D_{n l}^{\max } \geq 0$, expression (3) can be expressed as $D_{n l}(t+1)=\min \left[\max \left[D_{n l}(t)-a_{n l}(t), 0\right]+\right.$ $\left.\lambda_{n l}(t), D_{n l}^{\max }\right]$ for all $n \in \mathcal{N}$ and $l \in \mathcal{L}(n)$.
} 
input rates (i.e, input rates can be inside or outside of the network capacity region).

Furthermore, at the network layer each BS maintains a set of internal queues for storing current backlog (or unfinished work) of its users. Let $Q_{n l}(t)$ represents the current backlog of $l$ th user in $n$th BS. Then the evolution of the size of $Q_{n l}(t)$ is given by [23]

$$
Q_{n l}(t+1)=\max \left[Q_{n l}(t)-r_{n l}(t), 0\right]+a_{n l}(t),
$$

for all $n \in \mathcal{N}$ and $l \in \mathcal{L}(n)$, where $r_{n l}(t)$ is the transmission rate (defined in (2)) offered to $l$ th user of $n$th BS during time slot $t$. Here, we adopt the notion of strong stability, ${ }^{5}$ and we say that the network is strongly stable if ${ }^{6}$ [23], [29]

$$
\bar{Q}_{n l} \triangleq \limsup _{t \rightarrow \infty} \frac{1}{t} \sum_{\tau=1}^{t} \mathrm{E}\left\{Q_{n l}(\tau)\right\}<\infty, n \in \mathcal{N}, l \in \mathcal{L}(n),
$$

where the expectation depends on the control policy, and is with respect to the random channel states and the control actions made in reaction to these channel states. ${ }^{7}$ Intuitively, expression (5) means that a queue is strongly stable if its time average backlog is finite; and a network is strongly stable if all individual queues of the network are strongly stable.

At each time slot, for $l$ th user of $n$th BS the network controller admits $a_{n l}(t)$ data into the internal queue for transmission. Note that under network stability, admitted data $a_{n l}(t)$ for all $t$ in the internal queue is transmitted to the corresponding user over a finite period of time [29]. Thus, we define an utility of the user in terms of admitted data rate $a_{n l}(t)$, instead of transmission rate $r_{n l}(t)$. To define the utility of $l$ th user of BS $n$, let $\bar{a}_{n l}(t)$ denote the admitted time average rate up to time slot $t$, i.e., $\bar{a}_{n l}(t) \triangleq \frac{1}{t} \sum_{\tau=1}^{t} \mathrm{E}\left\{a_{n l}(\tau)\right\}$. Then, associated with each user, we define a non-decreasing concave utility function $g_{n l}\left(\bar{a}_{n l}(t)\right)$. The utility function $g_{n l}\left(\bar{a}_{n l}(t)\right)$ represents a monetary measure of the satisfaction that $n$th operator receives by sending data to its $l$ th user based on its current data rate $\bar{a}_{n l}(t)$. Finally, we define the time average expected profit of $n$th operator as

$$
\begin{aligned}
\bar{U}_{n} \triangleq & \liminf _{t \rightarrow \infty}\left(\sum_{l \in \mathcal{L}(n)} g_{n l}\left(\bar{a}_{n l}(t)\right)\right. \\
& +\frac{1}{t} \sum_{\tau=1}^{t} \mathrm{E}\left\{q_{n}(\tau)\left(\sum_{s \in \mathcal{S}(\underline{n}, \tau)} w_{s}-B\right)^{+}\right\} \\
& \left.-\frac{1}{t} \sum_{\tau=1}^{t} \mathrm{E}\left\{q_{\underline{n}}(\tau)\left(\sum_{s \in \mathcal{S}(n, \tau)} w_{s}-B\right)^{+}\right\}\right),
\end{aligned}
$$

where the second right hand term in (6) represents an amount that $n$th operator gets by leasing its spectrum band to its opponent; and the third right hand term represents an amount that $n$th operator pays to its opponent for renting the extra spectrum band. Note that during any given time slot, only one operator

\footnotetext{
${ }^{5}$ A definition of strong stability is general, and it also implies other forms of stability [29, Th. 2.8].

${ }^{6}$ Note that we use a commonly used procedure, and express the long term average as the time average of expectation, which leads to a tractable algorithm [35]-[41].

${ }^{7}$ Throughout the paper, all expectation are taken with respect to the random channel states and the control actions made in reaction to these channel states, unless stated otherwise.
}

can use more than $B \mathrm{~Hz}$ from the spectrum pool $2 B \mathrm{~Hz}$. Hence, during any given time slot, operators either lease or rent a portion of the common spectrum band. Specifically, during time slot $\tau$, either term $q_{n}(\tau)\left(\sum_{s \in \mathcal{S}(\underline{n}, \tau)} w_{s}-B\right)^{+}$or term $q_{\underline{n}}(\tau)\left(\sum_{s \in \mathcal{S}(n, \tau)} w_{s}-B\right)^{+}$is nonzero.

\section{Problem Formulation}

Our objective is to maximize the gain in profits of both operators by sharing their licensed spectrum bands with each other, rather than using them exclusively. Furthermore, we want to distribute the surplus (i.e., the increase in the profit) fairly among the operators, so that they have an incentive to share their licensed spectrum with each other. To do this, we model the spectrum sharing between two operators as a two-person bargaining problem [25]-[28] and cast as a stochastic optimization problem.

Let $U_{n}^{0}$ denotes the utility gain of $n$th operator that it gets before sharing its spectrum band with the other operator. In the context of bargaining problem, the utility $U_{n}^{0}$ is commonly known as a disagreement point, and it is assumed to be known. We assume that each operator knows a value of $U_{n}^{0}$ with their past experience. Then the benefits of the operators obtained by sharing their license spectrum bands with each other is $\bar{U}_{n}-U_{n}^{0}$ for all $n \in \mathcal{N}$. For tractability, we assume that a per-unit price of the spectrum band set by each operator is bounded, i.e., $0 \leq q_{n}(t) \leq q^{\max }$ for all $n \in \mathcal{N}$. Then the optimization problem to maximize the gain in operators profits, fairly ${ }^{8},{ }^{9}$ subject to the network stability and the maximum power constraint for each BS can be expressed as

$$
\begin{array}{ll}
\operatorname{maximize} & \sum_{n \in \mathcal{N}} \log \left(\bar{U}_{n}-U_{n}^{0}\right) \\
\text { subject to } & \bar{U}_{n} \geq U_{n}^{0}, \quad n \in \mathcal{N} \\
& \bar{Q}_{n l}<\infty, \quad n \in \mathcal{N}, l \in \mathcal{L}(n) \\
& 0 \leq q_{n}(t) \leq q^{\max }, \quad n \in \mathcal{N}, \forall t \\
& \sum_{l \in \mathcal{L}(n)} \sum_{s \in \mathcal{S}(n, t)}\left\|\mathbf{m}_{n l, s}(t)\right\|_{2}^{2} \leq p_{n}^{\max }, n \in \mathcal{N}, \forall t \\
& \mathcal{S}(1, t) \cap \mathcal{S}(2, t)=\emptyset, \quad \mathcal{S}(1, t), \mathcal{S}(2, t) \subseteq \mathcal{S}, \forall t,
\end{array}
$$

with variables $\left\{q_{n}(t), \mathcal{S}(n, t)\right\}_{n \in \mathcal{N}}, \quad\left\{a_{n l}(t)\right\}_{n \in \mathcal{N}, l \in \mathcal{L}(n)}$, and $\left\{\mathbf{m}_{n l, s}(t)\right\}_{n \in \mathcal{N}, l \in \mathcal{L}(n), s \in \mathcal{S}}$ for all $t \in\{1,2, \ldots\}$; where $\bar{U}_{n}$ and $\bar{Q}_{n l}$ are defined in (6) and (5), respectively. The constraint (7a) ensures that the profits of the operators obtained by sharing their spectrum bands are greater than without sharing their spectrum bands with each other. The constraint (7b) ensures that the network is stable. The constraint (7d) limits the total transmit power of each $\mathrm{BS}$, and constraint (7e) ensures that a subchannel is allocated only to a single operator.

\footnotetext{
${ }^{8}$ For $U_{n}^{0}=0$ for all $n \in \mathcal{N}$, problem (7) is a proportional fair utility maximization problem [27], [29, Ch. 5], [42]. Thus, the objective function of problem (7) is a generalized proportional fairness objective [17], [43].

${ }^{9}$ Efficient utilization of the common spectrum pool can be obtained by maximizing the social welfare objective $\sum_{n \in \mathcal{N}} \bar{U}_{n}$, without regards to the spectrum prices $\left\{q_{n}\right\}_{n \in \mathcal{N}}$ because the payment will be canceled out. However, the maximization of the social welfare objective may not ensure the fairness in operator profits. In co-primary spectrum access, both operators want to maximize their profit, as both operators put their licensed spectrum band in the common spectrum pool. In other word, a fair distribution in the operators' profit is desirable.
} 


\section{Dynamic Algorithm Via Lyapunov Optimization}

In this section we use the cross-layer stochastic optimization framework of [23] and [29] to solve problem (7). We start by transforming problem (7) such that it conform to the structure required for the drift-plus-penalty method of [23] and [29]. Then, we apply the drift-plus-penalty minimization method to the transformed problem to derive the steps of the proposed dynamic control algorithms.

\section{A. Transformed Problem via Auxiliary Variables}

We start by equivalently reformulating problem (7) by introducing an auxiliary variable $\bar{\mu}_{n}$, for all $n \in \mathcal{N}$, as

$$
\begin{array}{ll}
\operatorname{maximize} & \sum_{n \in \mathcal{N}} \log \left(\bar{\mu}_{n}\right) \\
\text { subject to } & \bar{\mu}_{n} \leq \bar{U}_{n}-U_{n}^{0}, \quad n \in \mathcal{N} \\
& \text { constraints }(7 a)-(7 e),
\end{array}
$$

with variables $\left\{\bar{\mu}_{n}\right\}_{n \in \mathcal{N}}, \quad\left\{q_{n}(t)\right\}_{n \in \mathcal{N}}, \quad\left\{a_{n l}(t)\right\}_{n \in \mathcal{N}, l \in \mathcal{L}(n)}$, $\left\{\mathbf{m}_{n l, s}(t)\right\}_{n \in \mathcal{X}, l \in \mathcal{L}(n), s \in \mathcal{S}}, \mathcal{S}(1, t)$, and $\mathcal{S}(2, t)$ for all $t \in$ $\{1,2, \ldots\}$. Note that the first inequality constraints of problem (8) holds with equality at the optimal solution due to a monotonic increasing property of the objective function.

To use the drift-plus-penalty minimization method [23], [29] for problem (8), we now assume that the auxiliary variable $\bar{\mu}_{n}$ is a time average of auxilary variables $\mu_{n}(t)$ for all $t=\{1,2, \ldots\}$, i.e., $\bar{\mu}_{n} \triangleq \lim _{t \rightarrow \infty} \frac{1}{t} \sum_{\tau=1}^{t} \mathrm{E}\left\{\mu_{n}(\tau)\right\}$. Then by following the approach of [29, Ch. 5.0.5], we modify problem (8) as the following optimization problem:

$$
\begin{array}{ll}
\operatorname{maximize} & \sum_{n \in \mathcal{N}} \overline{\log \left(\mu_{n}\right)} \\
\text { subject to } & \bar{\mu}_{n} \leq \bar{U}_{n}-U_{n}^{0}, \quad n \in \mathcal{N} \\
& \bar{U}_{n} \geq U_{n}^{0}, \quad n \in \mathcal{N} \\
& \bar{Q}_{n l}<\infty, \quad n \in \mathcal{N}, l \in \mathcal{L}(n) \\
& \text { constraints }(7 c)-(7 e),
\end{array}
$$

with the optimization variables $\left\{\mu_{n}(t)\right\}_{n \in \mathcal{N}},\left\{q_{n}(t)\right\}_{n \in \mathcal{N}}$, $\left\{a_{n l}(t)\right\}_{n \in \mathcal{N}, l \in \mathcal{L}(n)}, \mathcal{S}(1, t), \mathcal{S}(2, t)$, and $\left\{\mathbf{m}_{n l, s}(t)\right\}_{n \in \mathcal{N}, l \in \mathcal{L}(n), s \in \mathcal{S}}$ for all $t \in\{1,2, \ldots\}$; where $\overline{\log \left(\mu_{n}\right)}$ is defined as

$$
\overline{\log \left(\mu_{n}\right)} \triangleq \lim _{t \rightarrow \infty} \frac{1}{t} \sum_{\tau=1}^{t} \mathrm{E}\left\{\log \left(\mu_{n}(\tau)\right)\right\} .
$$

Note that by using Jensens inequality we can easily verify that $\overline{\log \left(\mu_{n}\right)}$ is lower bounds on $\log \left(\bar{\mu}_{n}\right)$. Thus the solution of problem (9) is also feasible for the original problem (7), and hence problem (9) provides reasonable lower bound for the original problem (7).

\section{B. Solving the Transformed Problem}

In this section we use the drift-plus-penalty minimization method introduced in [23] and [29] to solve problem (9). In the drift-plus-penalty minimization method, the inequality constrains (9a) and (9b) are enforced by transforming them into a queue stability problem. In other words, for each inequality constraint, in (9a) and (9b), a virtual queue is introduced in such a way that the stability of these virtual queues implies the feasibility of constraints (9a) and (9b).
Let $\left\{X_{n}(t)\right\}_{n \in \mathcal{N}}$ be virtual queues associated with constraint (9a). We update the virtual queue $X_{n}(t)$ for all $n \in \mathcal{N}$ at each time slot as

$$
X_{n}(t+1)=\max \left[X_{n}(t)-x_{n}^{\text {out }}(t), 0\right]+x_{n}^{\text {in }}(t),
$$

where,

$$
\begin{aligned}
x_{n}^{\text {out }}(t) & =\sum_{l \in \mathcal{L}(n)} g_{n l}\left(a_{n l}(t)\right)+q_{n}(t)\left(\sum_{s \in \mathcal{S}(\underline{n}, t)} w_{s}-B\right)^{+}, \\
x_{n}^{\text {in }}(t) & =\mu_{n}(t)+U_{n}^{0}+q_{\underline{n}}(t)\left(\sum_{s \in \mathcal{S}(n, t)} w_{s}-B\right)^{+} .
\end{aligned}
$$

Note that $X_{n}(t)$ can be viewed as a backlog in a virtual queue with input rate $x_{n}^{\text {in }}(t)$ and service rate $x_{n}^{\text {out }}(t)$. If virtual queues $\left\{X_{n}(t)\right\}_{n \in \mathcal{N}}$ are strongly stable (see expression (5) for the definition of strong stability), then constraint (9a) is satisfied [23, Sec. 5.4.1].

Likewise, to ensure inequality constraint (9b), we define virtual queues $\left\{Y_{n}(t)\right\}_{n \in \mathcal{N}}$; and update $Y_{n}(t)$ for all $n \in \mathcal{N}$ according to the following dynamics:

$$
Y_{n}(t+1)=\max \left[Y_{n}(t)-y_{n}^{\text {out }}(t), 0\right]+y_{n}^{\text {in }}(t),
$$

where,

$$
\begin{aligned}
y_{n}^{\text {out }}(t) & =\sum_{l \in \mathcal{L}(n)} g_{n l}\left(a_{n l}(t)\right)+q_{n}(t)\left(\sum_{s \in \mathcal{S}(\underline{n}, t)} w_{s}-B\right)^{+}, \\
y_{n}^{\text {in }}(t) & =U_{n}^{0}+q_{\underline{n}}(t)\left(\sum_{s \in \mathcal{S}(n, t)} w_{s}-B\right)^{+} .
\end{aligned}
$$

Stability of virtual queues $\left\{Y_{n}(t)\right\}_{n \in \mathcal{N}}$ ensures constraint (9b).

We now define Lyapunov function and its drift, which will be used to define a queue stability problem for the actual queues $\left\{Q_{n l}(t)\right\}_{n \in \mathcal{N}, l \in \mathcal{L}(n)}$ and the virtual queues $\left\{X_{n}(t), Y_{n}(t)\right\}_{n \in \mathcal{X} \text {. For }}$ a compact representation, let us use $\Theta(t)$ to denote a vector of the actual and the virtual queues, i.e., $\Theta(t)=\left[Q_{11}(t), \ldots, Q_{2 L_{2}}(t), X_{1}(t), X_{2}(t), Y_{1}(t), Y_{2}(t)\right]^{\mathrm{T}}$. Then we define a quadratic Lyapunov function $L(\Theta(t))$ as [23], [29]

$$
\begin{aligned}
L(\Theta(t))=\frac{1}{2}[ & \sum_{n \in \mathcal{N}} \sum_{l \in \mathcal{L}(n)} Q_{n l}(t)^{2} \\
& \left.+\sum_{n \in \mathcal{N}} X_{n}(t)^{2}+\sum_{n \in \mathcal{N}} Y_{n}(t)^{2}\right] .
\end{aligned}
$$

The Lyapunov function $L(\Theta(t))$ is a scalar measure of network congestion. Intuitively, if $L(\Theta(t))$ is small then all queues are small; and if $L(\Theta(t))$ is large then at least one queue is large. Thus, by minimizing a drift in the Lyapunov function (i.e., by minimizing a difference in the Lyapunov function from one slot to the next) queues $\left\{Q_{n l}(t)\right\}_{n \in \mathcal{K}, l \in \mathcal{L}(n)}$ and $\left\{X_{n}(t), Y_{n}(t)\right\}_{n \in \mathcal{N}}$ can be stabilized [29]. By using expression (17), the drift in the Lyapunov function (i.e., the expected change in the Lyapunov function from one slot to the next) can be written as

$$
\Delta(\Theta(t)) \triangleq \mathrm{E}\{L(\Theta(t+1))-L(\Theta(t)) \mid \Theta(t)\} .
$$

We now use the drift-plus-penalty minimization method introduced in [23] and [29] to solve problem (9). In this method, a control policy that solves problem (9) is obtained by 
minimizing an upper bound on the following drift-plus-penalty expression [23], [29]:

$$
\Delta(\Theta(t))-V \sum_{n \in \mathcal{N}} \mathrm{E}\left\{\log \left(\mu_{n}(t)\right) \mid \Theta(t)\right\},
$$

where $V \geq 0$, subject to the constraint (9d) in each time slot, i.e.,

$$
\begin{aligned}
& 0 \leq q_{n}(t) \leq q^{\max }, \quad n \in \mathcal{N} \\
& \sum_{l \in \mathcal{L}(n)} \sum_{s \in \mathcal{S}(n, t)}\left\|\mathbf{m}_{n l, s}(t)\right\|_{2}^{2} \leq p_{n}^{\max }, \quad n \in \mathcal{N} \\
& \mathcal{S}(1, t) \cap \mathcal{S}(2, t)=\emptyset, \quad \mathcal{S}(1, t), \mathcal{S}(2, t) \subseteq \mathcal{S} .
\end{aligned}
$$

Note that the expression (19) has two terms. The first term is the drift $\Delta(\Theta(t))$; and in [23] and [29] it is shown that by minimizing the drift $\Delta(\Theta(t))$, at each time slot, we can satisfy inequality constrains (9a)-(9c). The second term is $-\sum_{n \in \mathcal{N}} \mathrm{E}\left\{\log \left(\mu_{n}(t)\right) \mid \Theta(t)\right\}$, and by minimizing it at each time slot, the objective of problem (9) is maximized. Thus, by varying parameter $V$ we can obtain a desired trade-off between the size of the queue backlogs and the profits of the operators.

In the rest of this section, to simplify algorithm development, we first find an upper bound of expression (19). Then we present a dynamic control algorithm to solve problem (9) that, at each time slot, minimizes the upper bound of expression (19) subject to constraints (20)-(22).

To obtain an upper bound of expression (19), by using expressions (4), (11), and (14), we note that ${ }^{10}$

$$
\begin{aligned}
Q_{n l}(t+1)^{2} \leq & Q_{n l}(t)^{2}+a_{n l}(t)^{2}+r_{n l}(t)^{2} \\
& +2 Q_{n l}(t)\left[a_{n l}(t)-r_{n l}(t)\right], \\
X_{n}(t+1)^{2} \leq & X_{n}(t)^{2}+x_{n}^{\text {in }}(t)^{2}+x_{n}^{\text {out }}(t)^{2} \\
& +2 X_{n}(t)\left[x_{n}^{\text {in }}(t)-x_{n}^{\text {out }}(t)\right], \\
Y_{n}(t+1)^{2} \leq & Y_{n}(t)^{2}+y_{n}^{\text {in }}(t)^{2}+y_{n}^{\text {out }}(t)^{2} \\
& +2 Y_{n}(t)\left[y_{n}^{\text {in }}(t)-y_{n}^{\text {out }}(t)\right] .
\end{aligned}
$$

Then, by using expressions (18) and inequalities (23)-(25), an upper bound of expression (19) can be expressed as

$$
\begin{aligned}
& \Delta(\boldsymbol{\Theta}(t))-V \sum_{n \in \mathcal{N}} \mathrm{E}\left\{\log \left(\mu_{n}(t)\right) \mid \boldsymbol{\Theta}(t)\right\} \leq D \\
& \quad-V \sum_{n \in \mathcal{N}} \mathrm{E}\left\{\log \left(\mu_{n}(t)\right) \mid \boldsymbol{\Theta}(t)\right\} \\
& \quad+\sum_{n \in \mathcal{N}} \sum_{l \in \mathcal{L}(n)} Q_{n l}(t) \mathrm{E}\left\{a_{n l}(t)-r_{n l}(t) \mid \Theta(t)\right\} \\
& \quad+\sum_{n \in \mathcal{N}} X_{n}(t) \mathrm{E}\left\{x_{n}^{\text {in }}(t)-x_{n}^{\text {out }}(t) \mid \Theta(t)\right\} \\
& \quad+\sum_{n \in \mathcal{N}} Y_{n}(t) \mathrm{E}\left\{y_{n}^{\text {in }}(t)-y_{n}^{\text {out }}(t) \mid \Theta(t)\right\},
\end{aligned}
$$

where $D$ is a finite positive constant that satisfies the following condition for all $t$ :

$$
\begin{aligned}
D \geq \frac{1}{2}[ & \sum_{n \in \mathcal{N}} \sum_{l \in \mathcal{L}(n)} \mathrm{E}\left\{a_{n l}(t)^{2}+r_{n l}(t)^{2} \mid \boldsymbol{\Theta}(t)\right\} \\
& +\sum_{n \in \mathcal{N}} \mathrm{E}\left\{x_{n}^{\text {in }}(t)^{2}+x_{n}^{\text {out }}(t)^{2} \mid \boldsymbol{\Theta}(t)\right\} \\
& \left.+\sum_{n \in \mathcal{N}} \mathrm{E}\left\{y_{n}^{\text {in }}(t)^{2}+y_{n}^{\text {out }}(t)^{2} \mid \boldsymbol{\Theta}(t)\right\}\right] .
\end{aligned}
$$

${ }^{10}$ To write inequalities (23)-(25), we have used the fact that $(\max [Q-$ $b, 0]+A)^{2} \leq Q^{2}+A^{2}+b^{2}+2 Q(A-b)$ for any $Q \geq 0, b \geq 0$, and $A \geq 0$.
Furthermore, by substituting expressions (12), (13), (15), and (16) in (26), we get

$$
\begin{aligned}
\Delta & (\Theta(t))-V \sum_{n \in \mathcal{N}} \mathrm{E}\left\{\log \left(\mu_{n}(t)\right) \mid \Theta(t)\right\} \leq D \\
& -V \sum_{n \in \mathcal{N}} \mathrm{E}\left\{\log \left(\mu_{n}(t)\right) \mid \Theta(t)\right\} \\
& +\sum_{n \in \mathcal{N}} \sum_{l \in \mathcal{L}(n)} Q_{n l}(t) \mathrm{E}\left\{a_{n l}(t)-r_{n l}(t) \mid \Theta(t)\right\} \\
& +\sum_{n \in \mathcal{N}} X_{n}(t) \mathrm{E}\left\{\mu_{n}(t) \mid \Theta(t)\right\} \\
& +\sum_{n \in \mathcal{N}} W_{n}(t) \mathrm{E}\left\{U_{n}^{0}+q_{\underline{n}}(t)\left(\sum_{s \in \mathcal{S}(n, t)} w_{s}-B\right)^{+} \mid \Theta(t)\right\} \\
& -\sum_{n \in \mathcal{N}} W_{n}(t) \mathrm{E}\left\{\sum_{l \in \mathcal{L}(n)} g_{n l}\left(a_{n l}(t)\right)\right. \\
& \left.+q_{n}(t)\left(\sum_{s \in \mathcal{S}(\underline{n}, t)} w_{s}-B\right)^{+} \mid \Theta(t)\right\},
\end{aligned}
$$

where $W_{n}(t)=X_{n}(t)+Y_{n}(t)$.

Finally, we summarize the steps of the proposed dynamic control algorithms based on the drift-plus-penalty minimization method [23], [29] to solve problem (9) in Algorithm 1. The proposed algorithms observes queue backlogs $\Theta(t)$

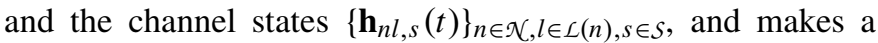
control action to minimize the righthand side of expression (28) subject to the constraints (20)-(22). The minimization of the righthand side of expression (28) can be decoupled across variables $q_{n}(t),\left\{a_{n l}(t)\right\}_{l \in \mathcal{L}(n)}, \mu_{n}(t)$, and $\left\{\left\{\mathbf{m}_{n l, s}(t)\right\}_{n \in \mathcal{N}, l \in \mathcal{L}(n), s \in \mathcal{S}(n, t)},\{S(n, t)\}_{n \in \mathcal{N}}\right\}$, resulting in subproblems as shown in Algorithm 1. Note that the drift-pluspenalty minimization method [23], [29] uses the concept of opportunistically minimizing an expectation [29, Ch. 1.8] to solve each subproblems.

In step 1 of Algorithm 1, the per-unit price of spectrum is set for the operators, and it is obtained by minimizing the righthand side of expression (28) over variables $\left\{q_{n}(t)\right\}_{n \in \mathcal{N}}$. Similar pricing strategy is obtained in [44], and it is known as bang-bang pricing. This pricing strategy alternates between periods of free service (i.e., price set to zero) and periods where price is set to a pre-specified maximum value $q^{\max }$, according to the values of virtual queues $\left\{W_{n}(t)=X_{n}(t)+\right.$ $\left.Y_{n}(t)\right\}_{n \in \mathcal{N}}$. The value of virtual queue $X_{n}(t)$ can be interpreted as the utility gain that $n$th operator has yet to obtain in order to maximize its profit. ${ }^{11}$ Thus the bang-bang pricing strategy, in step 1 , sets the price value to a pre-specified value $q^{\max 12}$ for an operator, whose utility is lagging behind than that of the other operator. Observe that except step 4 of Algorithm 1, the problems in each step of the algorithm are decoupled into two subproblems, one for each operator. In Section IV and Section V, we provide centralized and the distributed algorithms to solve step 4 of Algorithm 1, respectively. This leads to the centralized and the distributed versions

\footnotetext{
${ }^{11}$ Similarly, the value of virtual queue $Y_{n}(t)$ can be interpreted as the utility gain that $n$th operator has yet to obtain such that its utility becomes greater than its disagreement point $U_{n}^{0}$.

${ }^{12}$ In the bargaining framework, it is assumed that both operators have the full knowledge of the preferences of each other [25]. Thus, we assume that a value of $q^{\max }$ is set by the operators with their mutual agreement. For example, a value of $q^{\max }$ can be related to the utilities that operators can get by using their spectrum bands, i.e., we can set $q^{\max }=c \times g_{n l}\left(A^{\max }\right)$, for some constant $c>0$, (see step 2 of Algorithm 1 for a definition of $A^{\max }$ ).
} 
Algorithm 1: Algorithm for the Spectrum Sharing Problem (9)

1) Pricing: for each $n \in \mathcal{N}$, per-unit price $q_{n}(t)$ is chosen as

$$
q_{n}(t)= \begin{cases}q^{\max } & \text { if } W_{n}(t)>W_{\underline{n}}(t) \\ 0 & \text { otherwise. }\end{cases}
$$

2) Flow control: for each $n \in \mathcal{N}$, flow rate $a_{n l}(t)=a_{n l}$ for all $l \in \mathcal{L}(n)$, where $\left\{a_{n l}\right\}_{l \in \mathcal{L}(n)}$ solves the following optimization problem:

$$
\operatorname{maximize} W_{n}(t) \sum_{l \in \mathcal{L}(n)} g_{n l}\left(a_{n l}\right)-\sum_{l \in \mathcal{L}(n)} Q_{n l}(t) a_{n l}
$$$$
\text { subject to } 0 \leq a_{n l} \leq \min \left[D_{n l}(t), A^{\max }\right], \quad l \in \mathcal{L}(n),
$$

with variables $\left\{a_{n l}\right\}_{l \in \mathcal{L}(n)}$, where $A^{\max }>0$ is the algorithm parameter as described in [23, Sec. 4.2.1]. In the inequality constraint of problem (30), the term $D_{n l}(t)$ denotes the available data in the transport layer reservoir (see Section II-B).

3) Auxiliary variable: for each $n \in \mathcal{N}$, auxiliary variable $\mu_{n}(t)=\mu_{n}$, where $\mu_{n}$ solves the following optimization problem:

$$
\begin{aligned}
& \operatorname{maximize} V \log \left(\mu_{n}\right)-X_{n}(t) \mu_{n} \\
& \text { subject to } 0 \leq \mu_{n} \leq \mu^{\max },
\end{aligned}
$$

with variables $\mu_{n}$, where $\mu^{\max }>0$ is the algorithm parameter as described in [29, Ch. 5].

4) Resource allocation: solve the following optimization problem:

$$
\begin{aligned}
\operatorname{maximize} & \sum_{n \in \mathcal{N}} \sum_{l \in \mathcal{L}(n)} Q_{n l}(t) r_{n l}\left(S_{n}, \mathbf{m}_{n}\right) \\
& +\sum_{n \in \mathcal{K}} W_{n}(t) q_{n}(t)\left(\sum_{s \in \mathcal{S}(\underline{n})} w_{s}-B\right)^{+} \\
& -\sum_{n \in \mathcal{K}} W_{n}(t) q_{\underline{n}}(t)\left(\sum_{s \in \mathcal{S}(n)} w_{s}-B\right)^{+}
\end{aligned}
$$

subject to $\sum_{l \in \mathcal{L}(n)} \sum_{s \in \mathcal{S}(n)}\left\|\mathbf{m}_{n l, s}\right\|_{2}^{2} \leq p_{n}^{\max }, n \in \mathcal{N}$

$$
\mathcal{S}(1) \cap \mathcal{S}(2)=\emptyset, \quad \mathcal{S}(1), \mathcal{S}(2) \subseteq \mathcal{S},
$$

with variables $\left\{\mathbf{m}_{n l, s}\right\}_{n \in \mathcal{N}, l \in \mathcal{L}(n), s \in S(n)}$ and $\{S(n)\}_{n \in \mathcal{N}}$. Set $\mathbf{m}_{n l, s}(t)=\mathbf{m}_{n l, s}$ and $\mathcal{S}(n, t)=\mathcal{S}(n)$ for all $n \in \mathcal{N}$, $l \in \mathcal{L}(n)$, and $s \in \mathcal{S}(n)$.

5) Queue update: update $\left\{D_{n l}(t+1)\right\}_{n \in \mathcal{N}, l \in \mathcal{L}(n)},\left\{Q_{n l}(t+\right.$ 1) $\}_{n \in \mathcal{N}, l \in \mathcal{L}(n)},\left\{X_{n}(t+1)\right\}_{n \in \mathcal{N}}$, and $\left\{Y_{n}(t+1)\right\}_{n \in \mathcal{N}}$ by using expressions (3), (4), (11), and (14). Set $t=t+1$ and go to step 1.

of Algorithm 1 (i.e., the centralized and the distributed dynamic control algorithms). ${ }^{13}$

A performance of Algorithm 1 can be evaluated by using Theorem [23, Th. 5.4]. By using Theorem [23, Th. 5.4], we can show that Algorithm 1 yields the objective value of problem (7) and the network backlog with trade-off $[O(1 / V), O(V)]$. That is the objective value of problem (7) is pushed within $O(1 / V)$ of its maximum value, with an

\footnotetext{
${ }^{13}$ In the case when an operator deploys a heterogeneous system (for example, see [45]), the proposed Algorithm 1 can be extended by embedding the required inter-tier interference coordination strategy in the resource allocation subproblem (32). However, this extension falls outside the main scope of this paper, and it is left as an interesting future work.
}

increase in the network backlog with $V$. Derivation of this performance-backlog trade-off is omitted here due to space limitation.

\section{Resource Allocation - Centralized Algorithm}

In this section we focus on resource allocation problem (32). Problem (32) is a combinatorial problem, and it requires exponential complexity to find the global solution. Here, we derive a computational efficient and fast, but possibly suboptimal, algorithm for problem (32). The proposed algorithm is based on SCP [31].

We start by introducing binary variables $\left\{b_{n s}\right\}_{n \in \mathcal{N}, s \in \mathcal{S}}$ in problem (32). Variable $b_{n s}$ is set to one, if subchannel $w_{s}$ is assigned to $n$th operator, otherwise it is set to zero. Hence, by using binary variables $\left\{b_{n s}\right\}_{n \in \mathcal{N}, s \in \mathcal{S}}$, problem (32) can be equivalently written as

$$
\begin{aligned}
\operatorname{maximize} & \sum_{n \in \mathcal{N}} \sum_{l \in \mathcal{L}(n)} Q_{n l}(t) \sum_{s \in \mathcal{S}} b_{n s} w_{s} \log _{2}(1 \\
& \left.+\frac{\left|\mathbf{h}_{n l, s}^{\mathrm{H}}(t) \mathbf{m}_{n l, s}\right|^{2}}{b_{n s} N_{0} w_{s}+\sum_{j \in \mathcal{L}(n), j \neq l}\left|\mathbf{h}_{n l, s}^{\mathrm{H}}(t) \mathbf{m}_{n j, s}\right|^{2}}\right) \\
& +\sum_{n \in \mathcal{N}} W_{n}(t) q_{n}(t)\left(\sum_{s \in \mathcal{S}} b_{\underline{n} s} w_{s}-B\right)^{+} \\
& -\sum_{n \in \mathcal{N}} W_{n}(t) q_{\underline{n}}(t)\left(\sum_{s \in \mathcal{S}} b_{n s} w_{s}-B\right)^{+} \\
\text {subject to } & \sum_{l \in \mathcal{L}(n)} \sum_{s \in \mathcal{S}}\left\|\mathbf{m}_{n l, s}\right\|_{2}^{2} \leq p_{n}^{\max }, \quad n \in \mathcal{N} \\
& \sum_{n \in \mathcal{N}} b_{n s}=1, \quad s \in \mathcal{S} \\
& b_{n s}=\{0,1\}, \quad n \in \mathcal{N}, s \in \mathcal{S},
\end{aligned}
$$

with variables $\left\{b_{n s}\right\}_{n \in \mathcal{N}, s \in \mathcal{S}}$ and $\left\{\mathbf{m}_{n l, s}\right\}_{n \in \mathcal{N}, l \in \mathcal{L}(n), s \in \mathcal{S}}$; where $W_{n}(t)=X_{n}(t)+Y_{n}(t)$ for all $n \in \mathcal{N}$. Note that we have used expression (2) to write the objective function of problem (33), and variable $w_{s}$ is replaced with $b_{n s} w_{s}$. In problem (33) constraints (33b) and (33c) ensures that a subchannel $w_{s}$ is allocated to a single operator. Hence, the constraints associated with orthogonal subchannel allocation in problem (32) has been dropped out.

Now we relax hard binary constraint (33c), and employ a penalty function to promote binary value for variables $\left\{b_{n s}\right\}_{n \in \mathcal{K}, s \in \mathcal{S}}$, leading to

$$
\begin{aligned}
\operatorname{maximize} & \sum_{n \in \mathcal{N}} \sum_{l \in \mathcal{L}(n)} Q_{n l}(t) \sum_{s \in \mathcal{S}} b_{n s} w_{s} \log _{2}(1 \\
& \left.+\frac{\left|\mathbf{h}_{n l, s}^{\mathrm{H}}(t) \mathbf{m}_{n l, s}\right|^{2}}{b_{n s} N_{0} w_{s}+\left.\sum_{j \in \mathcal{L}(n), j \neq l} \mathbf{h}_{n l, s}^{\mathrm{H}}(t) \mathbf{m}_{n j, s}\right|^{2}}\right) \\
& +\sum_{n \in \mathcal{N}} W_{n}(t) q_{n}(t)\left(\sum_{s \in \mathcal{S}} b_{\underline{n} s} w_{s}-B\right)^{+} \\
& -\sum_{n \in \mathcal{N}} W_{n}(t) q_{\underline{n}}(t)\left(\sum_{s \in \mathcal{S}} b_{n s} w_{s}-B\right)^{+} \\
& +\delta \sum_{n \in \mathcal{N}} \sum_{s \in \mathcal{S}} b_{n s} \log \left(b_{n s}\right) \\
\text { subject to } & \sum_{l \in \mathcal{L}(n)} \sum_{s \in \mathcal{S}}\left\|\mathbf{m}_{n l, s}\right\|_{2}^{2} \leq p_{n}^{\max }, \quad n \in \mathcal{N} \\
& \sum_{n \in \mathcal{N}} b_{n s}=1, \quad s \in \mathcal{S} \\
& 0 \leq b_{n s} \leq 1, \quad n \in \mathcal{N}, s \in \mathcal{S},
\end{aligned}
$$


with variables $\left\{b_{n s}\right\}_{n \in \mathcal{N}, s \in \mathcal{S}}$ and $\left\{\mathbf{m}_{n l, s}\right\}_{n \in \mathcal{N}, l \in \mathcal{L}(n), s \in \mathcal{S}}$; where $\delta>0$ is a problem parameter. The penalty function $b_{n s} \log \left(b_{n s}\right)$ is the negative entropy function, and it has the maximum values at $b_{n s}$ equal to zero or one. Thus, there exits a value of parameter $\delta$ that can achieve binary values for variables $\left\{b_{n s}\right\}_{n \in \mathcal{N}, s \in \mathcal{S}}$. It is worth noting that problem (34) is a non-combinatorial optimization problem, however, it is still a nonconvex problem. In fact, problem (33) is NP-hard [30].

Since the RA problem is NP-hard, we use SCP to approximate its solution. In order to simplify the algorithm development, let us introduce variables $u_{n l, s}$ and $z_{n l, s}$ for all $n \in \mathcal{N}$, $l \in \mathcal{L}(n)$, and $s \in \mathcal{S}$ as

$$
\begin{aligned}
& u_{n l, s}=\sum_{j \in \mathcal{L}(n)}\left|\mathbf{h}_{n l, s}^{\mathrm{H}}(t) \mathbf{m}_{n j, s}\right|^{2}, \\
& z_{n l, s}=\sum_{j \in \mathcal{L}(n), j \neq l}\left|\mathbf{h}_{n l, s}^{\mathrm{H}}(t) \mathbf{m}_{n j, s}\right|^{2} .
\end{aligned}
$$

Furthermore, for the sake of brevity, let us define following functions:

$$
\begin{aligned}
\psi_{n l}\left(\mathbf{u}_{n l}, \mathbf{b}_{n}\right) & =-\sum_{s \in \mathcal{S}} b_{n s} w_{s} \log _{2}\left(N_{0} w_{s}+\frac{u_{n l, s}}{b_{n s}}\right) \\
\phi_{n l}\left(\mathbf{z}_{n l}, \mathbf{b}_{n}\right) & =-\sum_{s \in \mathcal{S}} b_{n s} w_{s} \log _{2}\left(N_{0} w_{s}+\frac{z_{n l, s}}{b_{n s}}\right) \\
\chi_{1}\left(\mathbf{b}_{1}\right) & =\left(W_{1}(t)-W_{2}(t)\right) q_{2}(t)\left(\sum_{s \in \mathcal{S}} b_{1 s} w_{s}-B\right)^{+} \\
\chi_{2}\left(\mathbf{b}_{2}\right) & =\left(W_{2}(t)-W_{1}(t)\right) q_{1}(t)\left(\sum_{s \in \mathcal{S}} b_{2 s} w_{s}-B\right)^{+} \\
\zeta\left(\mathbf{b}_{n}\right) & =\sum_{s \in \mathcal{S}} b_{n s} \log \left(b_{n s}\right)
\end{aligned}
$$

where $\mathbf{u}_{n l}=\left[u_{n l, 1}, \ldots, u_{n l, S}\right]^{\mathrm{T}}, \mathbf{z}_{n l}=\left[z_{n l, 1}, \ldots, z_{n l, S}\right]^{\mathrm{T}}$, and $\mathbf{b}_{n}=\left[b_{n 1}, \ldots, b_{n S}\right]^{\mathrm{T}}$. Then, by using expressions (35)-(40), and changing the sign of the objective function of problem (34), it can be equivalently expressed as the following minimization problem:

$$
\begin{aligned}
\operatorname{minimize} & \sum_{n \in \mathcal{N}}\left(\sum_{l \in \mathcal{L}(n)} Q_{n l}(t)\left(\psi_{n l}\left(\mathbf{u}_{n l}, \mathbf{b}_{n}\right)-\phi_{n l}\left(\mathbf{z}_{n l}, \mathbf{b}_{n}\right)\right)\right. \\
& \left.-\delta \zeta\left(\mathbf{b}_{n}\right)+\theta_{n}\right) \\
\text { subject to } & u_{n l, s}=\sum_{j \in \mathcal{L}(n)}\left|\mathbf{h}_{n l, s}^{\mathrm{H}}(t) \mathbf{m}_{n j, s}\right|^{2} \\
& n \in \mathcal{N}, l \in \mathcal{L}(n), s \in \mathcal{S} \\
& z_{n l, s}=\sum_{j \in \mathcal{L}(n), j \neq l}\left|\mathbf{h}_{n l, s}^{\mathrm{H}}(t) \mathbf{m}_{n j, s}\right|^{2} \\
& n \in \mathcal{N}, l \in \mathcal{L}(n), s \in \mathcal{S} \\
& \chi_{n}\left(\mathbf{b}_{n}\right) \leq \theta_{n}, \quad n \in \mathcal{N} \\
& \sum_{l \in \mathcal{L}(n)} \sum_{s \in \mathcal{S}}\left\|\mathbf{m}_{n l, s}\right\|_{2}^{2} \leq p_{n}^{\max }, n \in \mathcal{N} \\
& \sum_{n \in \mathcal{N}} b_{n s}=1, \quad s \in \mathcal{S} \\
& 0 \leq b_{n s} \leq 1, \quad n \in \mathcal{N}, s \in \mathcal{S}
\end{aligned}
$$

with variables $\left\{\mathbf{u}_{n l}, \mathbf{z}_{n l}\right\}_{n \in \mathcal{N}, l \in \mathcal{L}(n)}, \quad\left\{\mathbf{b}_{n}, \theta_{n}\right\}_{n \in \mathcal{N}}, \quad$ and $\left\{\mathbf{m}_{n l, s}\right\}_{n \in \mathcal{N}, l \in \mathcal{L}(n), s \in \mathcal{S}}$. Note that we have used the relation $\log (A / B)=\log (A)-\log (B)$ to express the objective function in problem (41). In the sequel, we first approximate problem (41) and express it as a difference of convex (DC) programming problem [46]. We then present an algorithm that finds a solution for DC problem (i.e., the solution for problem (41)) by solving a sequence of approximated convex problems.

Note that functions $\psi_{n l}\left(\mathbf{u}_{n l}, \mathbf{b}_{n}\right), \phi_{n l}\left(\mathbf{z}_{n l}, \mathbf{b}_{n}\right)$, and $\zeta\left(\mathbf{b}_{n}\right)$ are convex. Thus, the objective function of problem (41) can be expressed as the difference of the following two convex functions:

$$
\begin{aligned}
f_{0} & \triangleq f_{0}\left(\left\{\mathbf{u}_{n l}, \mathbf{b}_{n}, \theta_{n}\right\}_{n \in \mathcal{N}, l \in \mathcal{L}(n)}\right) \\
& =\sum_{n \in \mathcal{N}} \sum_{l \in \mathcal{L}(n)} Q_{n l}(t) \psi_{n l}\left(\mathbf{u}_{n l}, \mathbf{b}_{n}\right)+\theta_{n}, \\
g_{0} & \triangleq g_{0}\left(\left\{\mathbf{z}_{n l}, \mathbf{b}_{n}\right\}_{n \in \mathcal{N}, l \in \mathcal{L}(n)}\right) \\
& =\sum_{n \in \mathcal{N}} \sum_{l \in \mathcal{L}(n)} Q_{n l}(t) \phi_{n l}\left(\mathbf{z}_{n l}, \mathbf{b}_{n}\right)+\delta \zeta\left(\mathbf{b}_{n}\right),
\end{aligned}
$$

i.e., the objective function of problem (41) become $f_{0}-g_{0}$.

We now turn to the inequality constraint (41c). We can easily see that functions $\chi_{1}\left(\mathbf{b}_{1}\right)$ and $\chi_{2}\left(\mathbf{b}_{2}\right)$ are convex functions if $W_{1}(t) \geq W_{2}(t)$ and $W_{2}(t) \geq W_{1}(t)$, respectively. Otherwise, both functions $\chi_{1}\left(\mathbf{b}_{1}\right)$ and $\chi_{2}\left(\mathbf{b}_{2}\right)$ are concave functions. Thus, we introduce the following approximations for functions $\chi_{1}\left(\mathbf{b}_{1}\right)$ and $\chi_{2}\left(\mathbf{b}_{2}\right)$ :

$$
\begin{aligned}
& \hat{\chi}_{1}\left(\mathbf{b}_{1}\right) \\
& = \begin{cases}\left(W_{1}(t)-W_{2}(t)\right) q_{2}(t)\left(\sum_{s \in \mathcal{S}} b_{1 s} w_{s}-B\right)^{+} & W_{1}(t) \geq W_{2}(t) \\
\left(W_{1}(t)-W_{2}(t)\right) q_{2}(t)\left(\sum_{s \in \mathcal{S}} b_{1 s} w_{s}-B\right) & \text { otherwise, }\end{cases} \\
& \hat{\chi}_{2}\left(\mathbf{b}_{2}\right) \\
& = \begin{cases}\left(W_{2}(t)-W_{1}(t)\right) q_{1}(t)\left(\sum_{s \in \mathcal{S}} b_{2 s} w_{s}-B\right)^{+} & W_{2}(t) \geq W_{1}(t) \\
\left(W_{2}(t)-W_{1}(t)\right) q_{1}(t)\left(\sum_{s \in \mathcal{S}} b_{2 s} w_{s}-B\right) & \text { otherwise. }\end{cases}
\end{aligned}
$$

Note that in expressions (44) and (45), we have used the upper bound functions of $\chi_{1}\left(\mathbf{b}_{1}\right)$ and $\chi_{2}\left(\mathbf{b}_{2}\right)$ if $W_{1}(t)<W_{2}(t)$ and $W_{2}(t)<W_{1}(t)$, respectively.

To approximate (41a) and (41b) with convex constraints, let us introduce the new variable $\mathbf{M}_{n l, s}=\mathbf{m}_{n l, s} \mathbf{m}_{n l, s}^{\mathrm{H}}$ such that $\operatorname{Rank}\left(\mathbf{M}_{n l, s}\right)=1$ for all $n \in \mathcal{N}, l \in \mathcal{L}(n)$, and $s \in \mathcal{S}$. Then by applying a standard semidefinite relaxation technique (SDR) [47] and using expressions (42)-(45), problem (41) can be approximated as the following DC program:

$$
\begin{aligned}
\operatorname{minimize} & f_{0}\left(\left\{\mathbf{u}_{n l}, \mathbf{b}_{n}, \theta_{n}\right\}_{n \in \mathcal{N}, l \in \mathcal{L}(n)}\right) \\
& -g_{0}\left(\left\{\mathbf{z}_{n l}, \mathbf{b}_{n}\right\}_{n \in \mathcal{N}, l \in \mathcal{L}(n)}\right) \\
\text { subject to } & u_{n l, s}=\sum_{j \in \mathcal{L}(n)} \mathbf{h}_{n l, s}^{\mathrm{H}}(t) \mathbf{M}_{n j, s} \mathbf{h}_{n l, s}(t), \\
& n \in \mathcal{N}, l \in \mathcal{L}(n), s \in \mathcal{S} \\
& z_{n l, s}=\sum_{j \in \mathcal{L}(n), j \neq l} \mathbf{h}_{n l, s}^{\mathrm{H}}(t) \mathbf{M}_{n j, s} \mathbf{h}_{n l, s}(t), \\
& n \in \mathcal{N}, l \in \mathcal{L}(n), s \in \mathcal{S} \\
& \hat{\chi}_{n}\left(\mathbf{b}_{n}\right) \leq \theta_{n}, n \in \mathcal{N} \\
& \sum_{l \in \mathcal{L}(n)} \sum_{s \in \mathcal{S}} \operatorname{Trace}\left(\mathbf{M}_{n l, s}\right) \leq p_{n}^{\max }, n \in \mathcal{N} \\
& \sum_{n \in \mathcal{N}} b_{n s}=1, s \in \mathcal{S} \\
& 0 \leq b_{n s} \leq 1, n \in \mathcal{N}, s \in \mathcal{S} \\
& \mathbf{M}_{n l, s} \succeq 0, n \in \mathcal{N}, l \in \mathcal{L}(n), s \in \mathcal{S},
\end{aligned}
$$


with variables $\left\{\mathbf{u}_{n l}, \mathbf{z}_{n l}\right\}_{n \in \mathcal{N}, l \in \mathcal{L}(n), \quad\left\{\mathbf{b}_{n}, \theta_{n}\right\}_{n \in \mathcal{N},}, \quad \text { and }}$

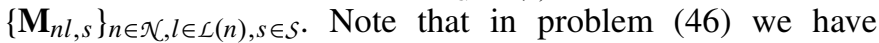
removed $\operatorname{Rank}\left(\mathbf{M}_{n l, s}\right)=1$ constraint for all $n \in \mathcal{N}, l \in \mathcal{L}(n)$, and $s \in \mathcal{S}$.

We find a solution for problem (46) by solving a sequence of approximated convex problems [46]. The best convex approximation of problem (46) can be obtained by replacing $g_{0}$ with its first order approximation (i.e., by replacing $\phi_{n l}\left(\mathbf{z}_{n l}, \mathbf{b}_{n}\right)$ and $\zeta\left(\mathbf{b}_{n}\right)$ with their first order approximations) [46]. The first order approximation of $\phi_{n l}\left(\mathbf{z}_{n l}, \mathbf{b}_{n}\right)$ near an arbitrary positive point $\left(\hat{\mathbf{z}}_{n l}, \hat{\mathbf{b}}_{n}\right)$ can be expressed as in (47), shown at the bottom of the page. The first order approximation of $\zeta\left(\mathbf{b}_{n}\right)$ near an arbitrary positive point $\hat{\mathbf{b}}_{n}$ can be expressed as

$$
\hat{\zeta}\left(\mathbf{b}_{n}\right)=\zeta\left(\hat{\mathbf{b}}_{n}\right)+\sum_{s \in \mathcal{S}}\left(1+\log \left(\hat{b}_{n s}\right)\right)\left(b_{n s}-\hat{b}_{n s}\right) .
$$

Hence, by using the expression of $f_{0}$ (see 42) and the first order approximation of $g_{0}$ (obtained by substituting (47) and (48) in (43)), problem (46) near an arbitrary positive point $\left(\hat{\mathbf{z}}_{n l}, \hat{\mathbf{b}}_{n}\right)$ can be expressed as the following convex optimization problem:

$$
\begin{gathered}
\operatorname{minimize} \sum_{n \in \mathcal{N}}\left(\sum_{l \in \mathcal{L}(n)} Q_{n l}(t)\left(\psi_{n l}\left(\mathbf{u}_{n l}, \mathbf{b}_{n}\right)-\hat{\phi}_{n l}\left(\mathbf{z}_{n l}, \mathbf{b}_{n}\right)\right)\right. \\
\left.-\delta \hat{\zeta}\left(\mathbf{b}_{n}\right)+\theta_{n}\right)
\end{gathered}
$$

subject to constraints $(46 a)-(46 g)$,

with variables $\left\{\mathbf{u}_{n l}, \mathbf{z}_{n l}\right\}_{n \in \mathcal{N}, l \in \mathcal{L}(n)}, \quad\left\{\mathbf{b}_{n}, \theta_{n}\right\}_{n \in \mathcal{N},}, \quad$ and $\left\{\mathbf{M}_{n l, s}\right\}_{n \in \mathcal{N}, l \in \mathcal{L}(n), s \in \mathcal{S} \text {. Finally, we summarize the proposed }}$ algorithm based on SCP for resource allocation problem (33) in Algorithm 2.

The first step in Algorithm 2 initializes the algorithm. Step 2 performs a first order approximation of functions $\hat{\phi}_{n l}\left(\mathbf{z}_{n l}, \mathbf{b}_{n}\right)$ and $\hat{\zeta}\left(\mathbf{b}_{n}\right)$ at the point $\left(\hat{\mathbf{z}}_{n l}, \hat{\mathbf{b}}_{n}\right)$ for all $n \in \mathcal{N}$ and $l \in \mathcal{L}(n)$. Then, the approximated convex problem (49) is solved at step 3. Step 4 checks the stopping criteria. ${ }^{14}$ Note that we have used an SDR technique to arrive at problem (49); thus a solution $\left\{\mathbf{M}_{n l, s}^{\star}\right\}_{n \in \mathcal{N}, l \in \mathcal{L}(n), s \in \mathcal{S}}$ obtained at step 3 may not be rank one in general. Hence, at step 5, we perform a rank one approximation of $\left\{\mathbf{M}_{n l, s}^{\star}\right\}_{n \in \mathcal{N}, l \in \mathcal{L}(n), s \in \mathcal{S}}$ to obtain the transmit beamformers $\left\{\mathbf{m}_{n l, s}^{\star}\right\}_{n \in \mathcal{N}, l \in \mathcal{L}(n), s \in \mathcal{S}}$ for problem (33). Specifically, we use randomization technique (randA method) presented in [49, Sec. IV] to obtain a rank one solution. Then the power and direction of the transmit beamformer associated with $l$ th user of BS $n$ in subchannel $s$ can be set to $p_{n l, s}=\left\|\mathbf{m}_{n l, s}^{\star}\right\|_{2}^{2}$ and $\mathbf{v}_{n l, s}=\mathbf{m}_{n l, s}^{\star} /\left\|\mathbf{m}_{n l, s}^{\star}\right\|_{2}$, respectively.

\footnotetext{
${ }^{14}$ The algorithm can be stopped either when a difference between the achieved objective value of problem (46) between two successive iterations is less than a given threshold, or it runs for a finite number of iterations [32, Sec. 3.2.2], [48, Sec. IV.B].
}

Algorithm 2: Centralized Algorithm for the Resource Allocation Problem (33)

1) Initialization: given initial feasible starting point $\left\{\mathbf{z}_{n l}^{0}, \mathbf{b}_{n}^{0}\right\}_{n \in \mathcal{N}, l \in \mathcal{L}(n)}$ and parameter $\delta>0$. Set iteration index $k=0$.

2) Set $\hat{\mathbf{z}}_{n l}=\mathbf{z}_{n l}^{k}$ and $\hat{\mathbf{b}}_{n}=\mathbf{b}_{n}^{k}$, then form $\hat{\phi}_{n l}\left(\mathbf{z}_{n l}, \mathbf{b}_{n}\right)$ and $\hat{\zeta}\left(\mathbf{b}_{n}\right)$ by using expressions (47) and (48), respectively, for all $n \in \mathcal{N}$ and $l \in \mathcal{L}(n)$.

3) Solve problem (49), and denote the solution by $\left\{\mathbf{u}_{n l}^{\star}, \mathbf{z}_{n l}^{\star}, \mathbf{b}_{n}^{\star}, \mathbf{M}_{n l, s}^{\star}\right\}_{n \in \mathcal{N}, l \in \mathcal{L}(n), s \in \mathcal{S}}$.

Update $\mathbf{u}_{n l}^{k+1}=\mathbf{u}_{n l}^{\star}, \mathbf{z}_{n l}^{k+1}=\mathbf{z}_{n l}^{\star}, \mathbf{b}_{n}^{k+1}=\mathbf{b}_{n}^{\star}$, and $\mathbf{M}_{n l, s}^{k+1}=\mathbf{M}_{n l, s}^{\star}$ for all $n \in \mathcal{N}, l \in \mathcal{L}(n)$, and $s \in \mathcal{S}$.

4) Stopping criterion: if the stopping criterion is satisfied, go to step 5. Otherwise set $k=k+1$, and go to step 2 .

5) Obtain a rank one approximation of $\mathbf{M}_{n l, s}^{\star}$ and denote it by $\mathbf{m}_{n l, s}^{\star}$, for all $n \in \mathcal{N}, l \in \mathcal{L}(n)$, and $s \in \mathcal{S}$. Return solution $\left\{\mathbf{b}_{n}^{\star}, \mathbf{m}_{n l, s}^{\star}\right\}_{n \in \mathcal{N}, l \in \mathcal{L}(n), s \in \mathcal{S}}$.

\section{A. Monotonic Convergence of Algorithm 2}

Algorithm 2 solves the DC programming problem (46) by using an approach similar to that in [46]. Hence Algorithm 2 is a descent algorithm [46, Sec. 1.3]. The proof is identical to that provided in [46, Sec. 1.3], and it is omitted here due to space limitation.

\section{Resource Allocation - Distributed Algorithm}

In this section we extend Algorithm 2 to derive a distributed algorithm for resource allocation problem (33). The distributed algorithm is derived by solving step 3 of Algorithm 2 (i.e., problem (49)) using ADMM [32].

We start by introducing an auxiliary variable $x_{n s}$ as a copy of $b_{n s}$ for all $n \in \mathcal{N}$ and $s \in \mathcal{S}$. Then problem (49) can be equivalently written as

$$
\begin{aligned}
\operatorname{minimize} & \sum_{n \in \mathcal{N}}\left(\sum_{l \in \mathcal{L}(n)} Q_{n l}(t)\left(\psi_{n l}\left(\mathbf{u}_{n l}, \mathbf{b}_{n}\right)-\hat{\phi}_{n l}\left(\mathbf{z}_{n l}, \mathbf{b}_{n}\right)\right)\right. \\
& \left.-\delta \hat{\zeta}\left(\mathbf{b}_{n}\right)+\theta_{n}\right)
\end{aligned}
$$

subject to constraints $(46 a)-(46 d),(46 f),(46 g)$

$$
\begin{aligned}
& b_{n s}=x_{n s}, \quad n \in \mathcal{N}, s \in \mathcal{S} \\
& \sum_{n \in \mathcal{N}} x_{n s}=1, \quad s \in \mathcal{S},
\end{aligned}
$$

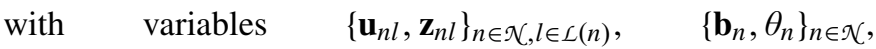
$\left\{\mathbf{M}_{n l, s}\right\}_{n \in \mathcal{N}, l \in \mathcal{L}(n), s \in \mathcal{S}}$, and $\left\{x_{n s}\right\}_{n \in \mathcal{N}, s \in \mathcal{S}}$. Observe that without constraint (50c), problem (50) can be easily decoupled into two subproblems, one for each operator.

$$
\begin{aligned}
\hat{\phi}_{n l}\left(\mathbf{z}_{n l}, \mathbf{b}_{n}\right)= & \phi_{n l}\left(\hat{\mathbf{z}}_{n l}, \hat{\mathbf{b}}_{n}\right)+\frac{1}{\log (2)} \sum_{s \in S}\left(-\frac{\hat{b}_{n s} w_{s}}{\hat{b}_{n s} N_{0} w_{s}+\hat{z}_{n l, s}}\right)\left(z_{n l, s}-\hat{z}_{n l, s}\right) \\
& +\frac{1}{\log (2)} \sum_{s \in S} w_{s}\left(\frac{\hat{z}_{n l, s}}{\hat{b}_{n s} N_{0} w_{s}+\hat{z}_{n l, s}}-\log \left(\frac{\hat{b}_{n s} N_{0} w_{s}+\hat{z}_{n l, s}}{\hat{b}_{n s}}\right)\right)\left(b_{n s}-\hat{b}_{n s}\right)
\end{aligned}
$$


We now express problem (50) more compactly. To do this, let us define the matrix $\mathbf{M}_{n l}=\left[\mathbf{M}_{n l, 1}, \ldots, \mathbf{M}_{n l, S}\right]$, and the following set:

$$
C_{n}=\left\{\begin{array}{l|l}
u_{n l, s}=\sum_{j \in \mathcal{L}(n)} \mathbf{h}_{n l, s}^{\mathrm{H}}(t) \mathbf{M}_{n j, s} \mathbf{h}_{n l, s}(t), \\
l \in \mathcal{L}(n), s \in \mathcal{S} \\
z_{n l, s}=\sum_{j \in \mathcal{L}(n), j \neq l} \mathbf{h}_{n l, s}^{\mathrm{H}}(t) \mathbf{M}_{n j, s} \mathbf{h}_{n l, s}(t), \\
\left\{\mathbf{u}_{n l}, \mathbf{z}_{n l},\right. \\
\left.\mathbf{M}_{n l}\right\}_{l \in \mathcal{L}(n)}, \\
\mathbf{b}_{n}, \theta_{n} \\
l \in \mathcal{L}(n), s \in \mathcal{S} \\
\hat{\chi}_{n}\left(\mathbf{b}_{n}\right) \leq \theta_{n} \\
\sum_{l \in \mathcal{L}(n)} \sum_{s \in \mathcal{S}} \operatorname{Trace}\left(\mathbf{M}_{n l, s}\right) \leq p_{n}^{\max } \\
0 \leq b_{n s} \leq 1, \quad s \in \mathcal{S} \\
\mathbf{M}_{n l, s} \succeq 0, \quad l \in \mathcal{L}(n), s \in \mathcal{S}
\end{array}\right\}
$$

Furthermore, for the sake of brevity, let us define the function $\Phi_{n}\left(\left\{\mathbf{u}_{n l}, \mathbf{z}_{n l}, \mathbf{M}_{n l}\right\}_{l \in \mathcal{L}(n)}, \mathbf{b}_{n}, \theta_{n}\right)$ as in (52), shown at the bottom of the page, and the following function:

$$
I\left(\left\{x_{n s}\right\}_{n \in \mathcal{N}, s \in \mathcal{S}}\right)= \begin{cases}0 & \sum_{n \in \mathcal{N}} x_{n s}=1 \text { for all } s \in \mathcal{S} \\ \infty & \text { otherwise. }\end{cases}
$$

Then by using expressions (51), (52), and (53), problem (50) can be written compactly as

$$
\begin{aligned}
& \operatorname{minimize} \sum_{n \in \mathcal{N}} \Phi_{n}\left(\left\{\mathbf{u}_{n l}, \mathbf{z}_{n l}, \mathbf{M}_{n l}\right\}_{l \in \mathcal{L}(n)}, \mathbf{b}_{n}, \theta_{n}\right) \\
&+I\left(\left\{x_{n s}\right\}_{n \in \mathcal{N}, s \in \mathcal{S}}\right) \\
& \text { subject to } b_{n s}=x_{n s}, \quad n \in \mathcal{N}, s \in \mathcal{S},
\end{aligned}
$$

with variables $\left\{\mathbf{u}_{n l}, \mathbf{z}_{n l}, \mathbf{M}_{n l}\right\}_{n \in \mathcal{N}, l \in \mathcal{L}(n)}, \quad\left\{\mathbf{b}_{n}, \theta_{n}\right\}_{n \in \mathcal{N}}$, and $\left\{x_{n s}\right\}_{n \in \mathcal{N}, s \in \mathcal{S}}$.

\section{A. ADMM Algorithm}

To derive the ADMM algorithm we first form the augmented Lagrangian [32] of problem (54). Let $\left\{\lambda_{n s}\right\}_{n \in \mathcal{N}, s \in \mathcal{S}}$ be the dual variables associated with the equality constraints of problem (54). Then the augmented Lagrangian can be written as in (55), shown at the bottom of the page. In (55), a penalty parameter $\rho>0$ adds the quadratic penalty to the standard Lagrangian $L_{0}$ for the violation of the equality constraints of problem (54).

Each iteration of ADMM algorithm consists of the following three steps (56)-(58), shown at the bottom of the page [32]. In (56)-(58) superscript $i$ is the iteration counter. Note that steps (56) and (58) are completely decentralized, and hence, can be carried out independently in parallel at each operator. Step (57) requires to gather the updated local variables $\left(\left\{\mathbf{u}_{n l}^{i+1}, \mathbf{z}_{n l}^{i+1}\right\}_{l \in \mathcal{L}(n)}, \mathbf{b}_{n}^{i+1}, \theta_{n}^{i+1}\right)$ and the dual variables $\left\{\lambda_{n s}^{i}\right\}_{s \in \mathcal{S}}$ from both operators. In the sequel we first explain, in detail, how to solve the ADMM step (57), and then simplify the above ADMM update-steps (56)-(58) into two update-steps. Then, we summarize the proposed ADMM based distributed algorithm.

The update $\left\{x_{n s}^{i+1}\right\}_{n \in \mathcal{N}, s \in \mathcal{S}}$ in (57) is a solution of the following optimization problem:

$$
\begin{aligned}
\operatorname{minimize} & \sum_{n \in \mathcal{N}} \sum_{s \in \mathcal{S}}\left(\lambda_{n s}^{i}\left(b_{n s}^{i+1}-x_{n s}\right)+(\rho / 2)\left(b_{n s}^{i+1}-x_{n s}\right)^{2}\right) \\
& +I\left(\left\{x_{n s}\right\}_{n \in \mathcal{N}, s \in \mathcal{S}}\right),
\end{aligned}
$$

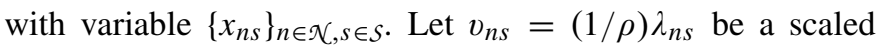
dual variable. Then by using notation (53), problem (59) can be equivalently expressed as

$$
\begin{aligned}
& \operatorname{minimize} \sum_{n \in \mathcal{N}} \sum_{s \in \mathcal{S}}(\rho / 2)\left(x_{n s}-v_{n s}^{i}-b_{n s}^{i+1}\right)^{2} \\
& \text { subject to } \sum_{n \in \mathcal{N}} x_{n s}=1, \quad s \in \mathcal{S},
\end{aligned}
$$

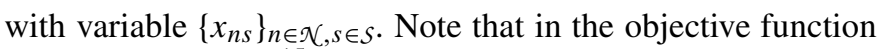
of problem (60), 15 we have dropped the constant term $(\rho / 2)\left(v_{n s}^{i}\right)^{2}$ since it does not effect the solution of the problem. For the equality constrained convex optimization problem (60), we can easily find the optimal solution by solving KKT optimality conditions [50, Ch. 5.5.3]. By solving KKT optimality conditions of problem (60), the solution $\left\{x_{n s}^{\star}\right\}_{n \in \mathcal{N}, s \in \mathcal{S}}$ can be

\footnotetext{
${ }^{15}$ We can simplify $\lambda_{n s}^{i}\left(b_{n s}^{i+1}-x_{n s}\right)+(\rho / 2)\left(b_{n s}^{i+1}-x_{n s}\right)^{2}=(\rho / 2)\left(x_{n s}-\right.$ $\left.v_{n s}^{i}-b_{n s}^{i+1}\right)^{2}-(\rho / 2)\left(v_{n s}^{i}\right)^{2}$.
}

$$
\begin{aligned}
& \Phi_{n}\left(\left\{\mathbf{u}_{n l}, \mathbf{z}_{n l}, \mathbf{M}_{n l}\right\}_{l \in \mathcal{L}(n)}, \mathbf{b}_{n}, \theta_{n}\right) \\
& =\left\{\begin{array}{l}
\sum_{l \in \mathcal{L}(n)} Q_{n l}(t)\left(\psi_{n l}\left(\mathbf{u}_{n l}, \mathbf{b}_{n}\right)-\hat{\phi}_{n l}\left(\mathbf{z}_{n l}, \mathbf{b}_{n}\right)\right)-\delta \hat{\zeta}\left(\mathbf{b}_{n}\right)+\theta_{n} \quad\left\{\mathbf{u}_{n l}, \mathbf{z}_{n l}, \mathbf{M}_{n l}\right\}_{l \in \mathcal{L}(n)}, \mathbf{b}_{n}, \theta_{n} \in \mathcal{C}_{n} \\
\infty \text { otherwise }
\end{array}\right. \\
& L_{\rho}\left(\left\{\mathbf{u}_{n l}, \mathbf{z}_{n l}, \mathbf{M}_{n l}, \mathbf{b}_{n}, \theta_{n}, x_{n s}, \lambda_{n s}\right\}_{n \in \mathcal{N}, l \in \mathcal{L}(n), s \in \mathcal{S}}\right)=\sum_{n \in \mathcal{N}} \Phi_{n}\left(\left\{\mathbf{u}_{n l}, \mathbf{z}_{n l}, \mathbf{M}_{n l}\right\}_{l \in \mathcal{L}(n)}, \mathbf{b}_{n}, \theta_{n}\right) \\
& +I\left(\left\{x_{n s}\right\}_{n \in \mathcal{N}, s \in \mathcal{S}}\right)+\sum_{n \in \mathcal{N}} \sum_{s \in \mathcal{S}}\left(\lambda_{n s}\left(b_{n s}-x_{n s}\right)+\frac{\rho}{2}\left(b_{n s}-x_{n s}\right)^{2}\right)
\end{aligned}
$$

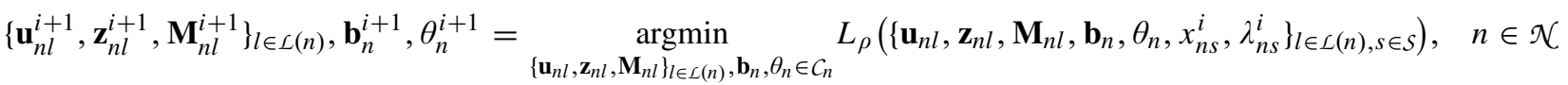

$$
\begin{aligned}
& \left\{x_{n s}^{i+1}\right\}_{n \in \mathcal{N}, s \in \mathcal{S}}=\underset{\left\{x_{n s}\right\}_{n \in \mathcal{N}, n \in \mathcal{S}}}{\operatorname{argmin}} L_{\rho}\left(\left\{\mathbf{u}_{n l}^{i+1}, \mathbf{z}_{n l}^{i+1}, \mathbf{M}_{n l}^{i+1}, \mathbf{b}_{n}^{i+1}, \theta_{n}^{i+1}, x_{n s}, \lambda_{n s}^{i}\right\}_{n \in \mathcal{N}, l \in \mathcal{L}(n), s \in \mathcal{S}}\right) \\
& \lambda_{n s}^{i+1}=\lambda_{n s}^{i}+\rho\left(b_{n s}^{i+1}-x_{n s}^{i+1}\right), \quad n \in \mathcal{N}, s \in \mathcal{S}
\end{aligned}
$$


expressed as

$$
x_{n s}^{\star}=v_{n s}^{i}+b_{n s}^{i+1}-\tilde{v}_{s}^{i}-\tilde{b}_{s}^{i+1}+1 / N,
$$

where $N=|\mathcal{N}|, \quad \tilde{v}_{s}^{i}=(1 / N) \sum_{n \in \mathcal{N}} v_{n s}^{i}$, and $\tilde{b}_{s}^{i+1}=$ $(1 / N) \sum_{n \in \mathcal{N}} b_{n s}^{i+1}$. Therefore, the update $x_{n s}^{i+1}$ is

$$
x_{n s}^{i+1}=x_{n s}^{\star}, \quad n \in \mathcal{N}, s \in \mathcal{S} .
$$

Now we substitute expression (61) for $x_{n s}^{i+1}$ in the dual variable update step (58). Furthermore, by using a scaled dual variable $v_{n s}=(1 / \rho) \lambda_{n s}$, step (58) can be simplified as

$$
v_{n s}^{i+1}=\tilde{v}_{s}^{i}+\tilde{b}_{s}^{i+1}-1 / N, \quad n \in \mathcal{N}, s \in \mathcal{S} .
$$

Expression (63) shows that the dual variables $v_{n s}$ for all $n \in \mathcal{N}$ are equal. Hence, the dual variables $\left\{v_{n s}\right\}_{n \in \mathcal{N}}$ can be replaced with a single variable $v_{s} \in \mathbb{R}$ in the ADMM iteration (56)-(58). Finally, by substituting the expression for $x_{n s}^{i}$ (that can be obtained from (62)) in (56), the ADMM iteration (56)-(58) can be simplified into the two steps (64) and (65), shown at the bottom of the page.

Note that we have used expression (55) to arrive at (64). By using notation (52), the optimization problem to update variables $\left(\left\{\mathbf{u}_{n l}^{i+1}, \mathbf{z}_{n l}^{i+1}, \mathbf{M}_{n l}^{i+1}\right\}_{l \in \mathcal{L}(n)}, \mathbf{b}_{n}^{i+1}, \theta_{n}^{i+1}\right)$ in (64) can be expressed as

$$
\begin{aligned}
\operatorname{minimize} & \sum_{l \in \mathcal{L}(n)} Q_{n l}(t)\left(\psi_{n l}\left(\mathbf{u}_{n l}, \mathbf{b}_{n}\right)-\hat{\phi}_{n l}\left(\mathbf{z}_{n l}, \mathbf{b}_{n}\right)\right) \\
& -\delta \hat{\zeta}\left(\mathbf{b}_{n}\right)+\theta_{n} \\
& +\sum_{s \in S}(\rho / 2)\left(b_{n s}-b_{n s}^{i}+\tilde{b}_{s}^{i}+v_{s}^{i}-1 / N\right)^{2}
\end{aligned}
$$

subject to $u_{n l, s}=\sum_{j \in \mathcal{L}(n)} \mathbf{h}_{n l, s}^{\mathrm{H}}(t) \mathbf{M}_{n j, s} \mathbf{h}_{n l, s}(t)$,

$$
\begin{aligned}
& l \in \mathcal{L}(n), s \in \mathcal{S} \\
& z_{n l, s}=\sum_{j \in \mathcal{L}(n), j \neq l} \mathbf{h}_{n l, s}^{\mathrm{H}}(t) \mathbf{M}_{n j, s} \mathbf{h}_{n l, s}(t), \\
& l \in \mathcal{L}(n), s \in \mathcal{S} \\
& \hat{\chi}_{n}\left(\mathbf{b}_{n}\right) \leq \theta_{n} \\
& \sum_{l \in \mathcal{L}(n)} \sum_{s \in \mathcal{S}} \operatorname{Trace}\left(\mathbf{M}_{n l, s}\right) \leq p_{n}^{\max } \\
& 0 \leq b_{n s} \leq 1, \quad s \in \mathcal{S} \\
& \mathbf{M}_{n l, s} \succeq 0, \quad l \in \mathcal{L}(n), s \in \mathcal{S},
\end{aligned}
$$

with variables $\left\{\mathbf{u}_{n l}, \mathbf{z}_{n l}\right\}_{l \in \mathcal{L}(n)}, \mathbf{b}_{n}, \theta_{n}$, and $\left\{\mathbf{M}_{n l, s}\right\}_{l \in \mathcal{L}(n), s \in \mathcal{S}}$. We now summarize the proposed distritbuted algorithm for the resource allocation problem (33) in Algorithm 3.

The steps of Algorithm 3 are similar to those of the centralized Algorithm 2, except the step 3 of both algorithms.
Algorithm 3: Distributed Algorithm for Problem (33)

1) Initialization: given initial feasible starting points $\left\{\mathbf{z}_{n l}^{0}, \mathbf{b}_{n}^{0}\right\}_{n \in \mathcal{N}, l \in \mathcal{L}(n)},\left\{v_{s}^{0}\right\}$, and parameters $\delta>0$ and $\rho>0$. Set iteration indices $i=0$ and $k=0$.

2) Set $\hat{\mathbf{z}}_{n l}=\mathbf{z}_{n l}^{i}$ and $\hat{\mathbf{b}}_{n}=\mathbf{b}_{n}^{i}$, then form $\hat{\phi}_{n l}\left(\mathbf{z}_{n l}, \mathbf{b}_{n}\right)$ and $\hat{\zeta}\left(\mathbf{b}_{n}\right)$ by using expressions (47) and (48), respectively, for all $n \in \mathcal{N}$ and $l \in \mathcal{L}(n)$.

3) ADMM iteration:

a) each operator $n \in \mathcal{N}$ updates the local variables $\left(\left\{\mathbf{u}_{n l}^{i+1}, \mathbf{z}_{n l}^{i+1}, \mathbf{M}_{n l}^{i+1}\right\}_{l \in \mathcal{L}(n)}, \mathbf{b}_{n}^{i+1}, \theta_{n}^{i+1}\right)$ by solving (66).

b) operators exchange their updated local variables $\left\{b_{n s}^{i+1}\right\}_{s \in \mathcal{S}}$ with each other.

c) each operator $n \in \mathcal{N}$ updates the dual variables $\left\{v_{s}^{i+1}\right\}_{s \in \mathcal{S}}$ by solving (65).

d) ADMM stopping criterion: if the stopping criterion is satisfied, go to step 4. Otherwise, set $i=i+1$, and go to step $3 \mathrm{a}$.

4) Stopping criterion: if the stopping criterion is satisfied, go to step 5 . Otherwise set $i=i+1, k=k+1$, and go to step 2 .

5) Set $\mathbf{b}_{n}^{\star}=\mathbf{b}_{n}^{i+1}$, obtain a rank one approximation of $\mathbf{M}_{n l, s}^{i+1}$ and denote it by $\mathbf{m}_{n l, s}^{\star}$, for all $n \in \mathcal{N}, l \in \mathcal{L}(n)$,

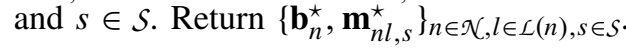

Step 3 of Algorithm 2 solves problem (49) in a central controller. However, in Algorithm 3 the same problem is solved distributively by using ADMM (i.e., by performing iterations in steps (3a)-(3d)). Note that we have applied ADMM to a convex problem, and therefore the ADMM iterations converge to the global optimal value [51, Prop. 4.2], [32]. Thus, by following Section IV-A, we can see that a sequence of the objective value of problem (46), that is produced upon ADMM convergence (i.e., after step 3 of Algorithm 3) is monotonic.

Step 3d of Algorithm 3 checks the ADMM stopping criteria. In ADMM algorithm, standard stopping criteria is to check the primal and dual residuals [32]. We refer to each execution of steps 2-4 as an outer iteration, and we use index $k$ to count it. Step 4 checks stopping criteria for the outer iteration. ${ }^{16}$

\footnotetext{
${ }^{16}$ The algorithm can be stopped either when a difference between the achieved objective value of problem (46) between two successive iterations is less than a given threshold, or it runs for a finite number of iterations [32, Sec. 3.2.2], [48, Sec. IV.B].
}

$$
\begin{aligned}
\left\{\mathbf{u}_{n l}^{i+1}, \mathbf{z}_{n l}^{i+1}, \mathbf{M}_{n l}^{i+1}\right\}_{l \in \mathcal{L}(n)}, \mathbf{b}_{n}^{i+1}= & \underset{\left\{\mathbf{u}_{n l}, \mathbf{z}_{n l}, \mathbf{M}_{n l}\right\}_{l \in \mathcal{L}(n)}, \mathbf{b}_{n} \in \mathcal{C}_{n}}{\operatorname{argmin}} \Phi_{n}\left(\left\{\mathbf{u}_{n l}, \mathbf{z}_{n l}, \mathbf{M}_{n l}\right\}_{l \in \mathcal{L}(n)}, \mathbf{b}_{n}\right) \\
& +\sum_{s \in \mathcal{S}}(\rho / 2)\left(b_{n s}-b_{n s}^{i}+\tilde{b}_{s}^{i}+v_{s}^{i}-1 / N\right)^{2}, \quad n \in \mathcal{N} \\
v_{s}^{i+1}= & v_{s}^{i}+\tilde{b}_{s}^{i+1}-1 / N, \quad s \in \mathcal{S}
\end{aligned}
$$




\section{B. Implementation of Algorithm 3}

Except step 3b all other steps of Algorithm 3 are decoupled over the operators. Hence all other steps, except step $3 b$, can be performed independently, in parallel. Step $3 \mathrm{~b}$ requires coordination between operators to exchange their updated value of local variables $\left\{b_{n s}^{i+1}\right\}_{s \in \mathcal{S}}$ for all $n \in \mathcal{N}$. Recall that $b_{n s}$ represents the local opinion of $n$th operator about the fraction of $s$ th subchannel that it uses. Since ADMM usually produces acceptable results for practical use within only a few iterations, a predefined or fixed number of iterations can be used as stopping criterion for the ADMM iterations [32] at step 3d. The transmit beamformers $\left\{\mathbf{m}_{n l, s}^{\star}\right\}_{n \in \mathcal{N}, l \in \mathcal{L}(n), s \in \mathcal{S}}$ for problem (33) are computed at step 5, upon the convergence of the algorithm. At step 5, we can use randA method ${ }^{17}$ presented in [49, Sec. IV] to obtain $\left\{\mathbf{m}_{n l, s}^{\star}\right\}_{n \in \mathcal{N}, l \in \mathcal{L}(n), s \in \mathcal{S}}$. Then the power and direction of the transmit beamformer associated with $l$ th user of BS $n$ in subchannel $s$ can be set to $p_{n l, s}=\left\|\mathbf{m}_{n l, s}^{\star}\right\|_{2}^{2}$ and $\mathbf{v}_{n l, s}=\mathbf{m}_{n l, s}^{\star} /\left\|\mathbf{m}_{n l, s}^{\star}\right\|_{2}$, respectively. It is worth to point out that, in Algorithm 3, operators do not need to share their users' data and the channel state information with each other.

\section{Early Termination of ADMM Iteration}

To speed up Algorithm 3 we can stop ADMM iteration after a finite number of iterations before it converges. In this case the intermediate solutions $\left\{b_{n s}^{i+1}\right\}_{n \in \mathcal{N}, s \in \mathcal{S}}$ provided by ADMM iteration do not necessarily result a feasible solution for the original problem (33). In particular constraint (33b) may not hold (i.e., $\sum_{n \in \mathcal{N}} b_{n s}^{i+1} \neq 1$ for some $s \in \mathcal{S}$ ). Thus, we need to project $\left\{b_{n s}^{i+1}\right\}_{n \in \mathcal{N}, s \in \mathcal{S}}$ on the set $\mathcal{F}$, defined as $\mathcal{F}=\left\{\left\{b_{n s}\right\}_{n \in \mathcal{N}, s \in \mathcal{S}} \mid \sum_{n \in \mathcal{N}} b_{n s}=1, s \in \mathcal{S}\right\}$, to evaluate expressions $\hat{\phi}_{n l}\left(\mathbf{z}_{n l}, \mathbf{b}_{n}\right)$ and $\hat{\zeta}\left(\mathbf{b}_{n}\right)$ at step 2 of Algorithm 3. Projection of $\left\{b_{n s}^{i+1}\right\}_{n \in \mathcal{N}, s \in \mathcal{S}}$ on the set $\mathcal{F}$ can be obtained by solving problem (60), with $v_{n s}^{i}$ set to zero for all $n \in \mathcal{N}$ and $s \in \mathcal{S}$. Hence the projection of $\left\{b_{n s}^{i+1}\right\}_{n \in \mathcal{N}, s \in \mathcal{S}}$ on the set $\mathcal{F}$ is $\left\{b_{n s}^{i+1}-\tilde{b}_{s}^{i+1}+1 / N\right\}_{n \in \mathcal{N}, s \in \mathcal{S}}$, which is obtained by setting $v_{n s}^{i}=0$ for all $n \in \mathcal{N}$ and $s \in \mathcal{S}$ in expression (61).

\section{Complexity of Algorithm 2 and Algorithm 3}

Algorithm 2 and Algorithm 3 are iterative ${ }^{18}$ algorithms. Thus, we focus on characterizing their complexity per iteration. Note that both algorithms are solving convex problems at each iteration (i.e., problem (49) is solved in Algorithm 2, and problem (66) is solved in Algorithm 3). Thus, these problems can be efficiently solved by using interior-point method that relies on the Newton's method applied to a sequence of modified versions of the original problem [50, Ch. 11]. The complexity of solving a Newton step for problem (49) is $O\left(\left(L S\left(T^{2}+2\right)+N S+N+S\right)^{3}\right)$, where $L=\sum_{n \in \mathcal{N}} L_{n}$; and that of problem (66) is $O\left(\left(L_{n} S\left(T^{2}+2\right)+S+1\right)^{3}\right)$

\footnotetext{
${ }^{17}$ Dominant eigenvalue and corresponding eigenvector of $\mathbf{M}_{n l, s}^{i+1}$ can also be used to obtain a rank one approximation.

${ }^{18}$ It is important to point out that, in practice, the quality of the solution achieved within the first few iterations are more important than the asymptotic results, as we usually have time to perform only small number of iterations.
}

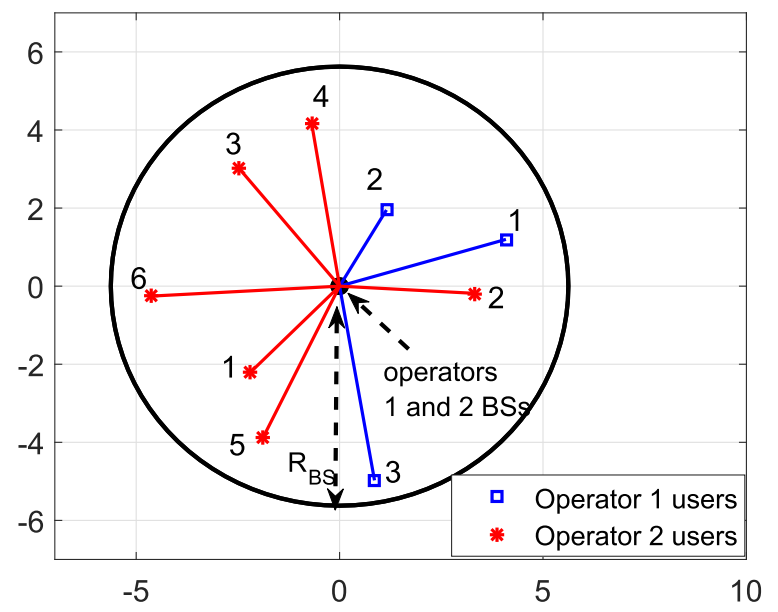

Fig. 2. A cell with two coexisting BSs belonging to different operators. BSs are placed on the same cell site. $\mathcal{N}=\{1,2\}, \mathcal{L}(1)=\{1,2,3\}$, and $\mathcal{L}(2)=\{1,2,3,4,5,6\}$.

[50, Ch. 10.4]. 19 Note that, in general, a convex problem requires only a modest number of Newton steps to solve it with high accuracy (i.e., a number of Newton steps between $30-100$ are enough for most of the applications) [50, Ch. 11.3.2].

\section{Simulation Results}

We illustrate the performance of the proposed Algorithm 1 by using the setup as shown in Fig. 2. The network consist of a cell with two coexisting BSs, belonging to two operators. The BSs are assumed to be installed in a same tower [52, Sec. 9], [53, Sec. 3], and they are placed at different height levels of the tower. ${ }^{20}$ Each BS consists of $T=2$ transmit antennas. We assume a circular cell, with a radius $R_{\mathrm{BS}}$. We assume that there are $L_{1}=3$ users associated with BS 1 , and $L_{2}=6$ users associated with BS 2. The locations of users associated with each BS are arbitrarily chosen as shown in Fig. 2. We assume that each operator shares $B=2 \mathrm{MHz}$ spectrum band, and hence, the total spectrum band of $4 \mathrm{MHz}$ is available for both operators. We split the spectrum band $4 \mathrm{MHz}$ into $S=4$ subchannels, and the bandwidth of each subchannel $w_{s}=1 \mathrm{MHz}$.

We assume an exponential path loss model, where the channel vector from $n$th BS to its $l$ th user on subchannel $s$ is modeled as $\mathbf{h}_{n l, s}(t)=\left(d_{n l}(t) / d_{0}\right)^{-\eta / 2} \mathbf{c}_{n l, s}(t)$, where $d_{n l}(t)$ is the distance from BS $n$ to its $l$ th user, $d_{0}$ is the far field reference distance [54], $\eta$ is the path loss exponent, and $\mathbf{c}_{n l, s}(t) \in \mathbb{C}^{T}$ is arbitrarily chosen from the distribution $\mathcal{C N}(0, \mathbf{I})$ (i.e., frequency-flat fading channel with uncorrelated antennas). Note that the term $\left(d_{n l}(t) / d_{0}\right)^{-\eta / 2}$ denotes large scale fading, and the term $\mathbf{c}_{n l, s}(t)$ denotes small scale fading.

\footnotetext{
${ }^{19}$ The complexity order are computed by relaxing the first and second equality constraints of problem (49) and (66), as they hold with equality at the optimal solution.

${ }^{20}$ This particular setup is chosen to illustrate that operators are operating in a same geographical area. However, our problem formulation and the proposed algorithms are general, and they are applicable when the BSs of the operators are far apart and also to the case of correlated channels.
} 


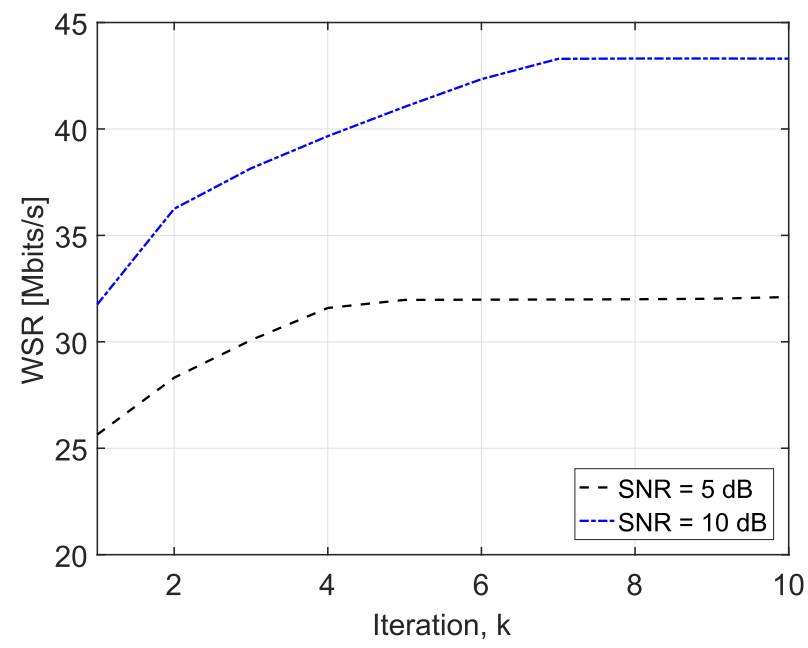

Fig. 3. WSR [Mbits/s] versus iteration for $\mathrm{SNR}=5 \mathrm{~dB}$ and $10 \mathrm{~dB}$ of Algorithm 2.

Here, we refer an arbitrarily generated set of fading coefficients $\check{C}(t)=\left\{\mathbf{c}_{n l, s}(t), d_{n l}(t) \mid n \in \mathcal{N}, l \in \mathcal{L}(n), s \in \mathcal{S}\right\}$ as a single fading realization.

We assume that $p_{n}^{\max }=p_{0}^{\max }$ for all $n \in \mathcal{N}$. We define the signal-to-noise ratio (SNR) operating point at a distance $R$ as $\operatorname{SNR}(R)=\left(R / d_{0}\right)^{-\eta} p_{0}^{\max } /\left(N_{0} w_{s}\right)$. In the following simulations, we set $d_{0}=1, \eta=4$, and the cell radius $R_{\mathrm{BS}}$ is fixed throughout the simulations such that $\operatorname{SNR}\left(R_{\mathrm{BS}}\right)=10 \mathrm{~dB}$ for $p_{0}^{\max } /\left(N_{0} w_{s}\right)=40 \mathrm{~dB}$.

To solve step 4 of Algorithm 1, we use either centralized Algorithm 2 or distributed Algorithm 3. Thus, we first present the performance of Algorithm 2 and Algorithm 3. Then, we evaluate the performance of Algorithm 1. In Algorithm 2 and Algorithm 3, we set penalty parameter $\delta=\left(0.1 w_{s}\right) k$. That is a varying penalty parameter $\delta$ is used such that more weight is given to the penalty function $\hat{\zeta}\left(\mathbf{b}_{n}\right)$ as algorithm progress. In Algorithm 3, for the ADMM iteration, we use the standard stopping criteria presented in [32, Sec. 3.3.1]. The stopping criteria in [32, Sec. 3.3.1] calculates the primal residual $r_{\text {pri }}^{i}=\left(\sum_{n \in \mathcal{N}} \sum_{s \in \mathcal{S}}\left(b_{n s}^{i}-x_{n s}^{i}\right)^{2}\right)^{1 / 2}$ and the dual residual $r_{\text {dual }}^{i}=\left(\rho \sum_{n \in \mathcal{N}} \sum_{s \in \mathcal{S}}\left(x_{n s}^{i}-x_{n s}^{i-1}\right)^{2}\right)^{1 / 2}$, see (61) for the expression of $x_{n s}^{i}$. Then ADMM iteration is stopped, if $r_{\text {pri }}^{i} \leq \epsilon$ and $r_{\text {dual }}^{i} \leq \epsilon$, where $\epsilon>0$ is a given tolerance. In the simulation, we set $\epsilon=0.1$. Furthermore, we limit the ADMM iteration to a maximum of 10 iterations.

To evaluate the performance of Algorithm 2 and Algorithm 3, we consider a single fading realization. The weight $Q_{n l}(t)$ associated with each user is set to one for all $n \in \mathcal{N}$ and $l \in \mathcal{L}(n)$; and the price per-unit spectrum $q_{n}(t)$ of each operator is set to zero for all $n \in \mathcal{N}$. As we set $q_{n}(t)=0$ for all $n \in \mathcal{N}$, Algorithm 2 and Algorithm 3 solve a weighted sum-rate (WSR) maximization problem (see problem (33)), and that jointly allocates spectrum band to the operators and design transmit beamformers.

Fig. 3 shows the convergence behavior of the centralized Algorithm 2 for $\mathrm{SNR}=5 \mathrm{~dB}$ and $\mathrm{SNR}=10 \mathrm{~dB}$. The WSR values of problem (33) are computed after step 3 of the algorithm. Results show that the proposed Algorithm 2 converges within the first few iterations.

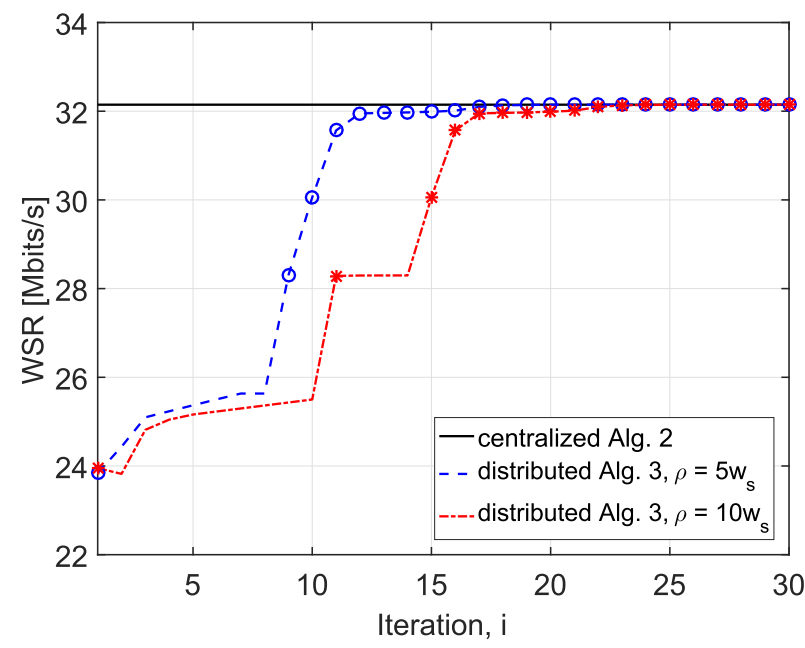

Fig. 4. WSR [Mbits/s] versus iteration for $\mathrm{SNR}=5 \mathrm{~dB}$ of Algorithm 3 .

Fig. 4 shows the convergence behavior of the distributed Algorithm 3 for $\mathrm{SNR}=5 \mathrm{~dB}$, along with the objective value obtained by the centralized Algorithm 2. We set the ADMM penalty parameter $\rho=5 w_{s}$ and $10 w_{s}$. The WSR values of problem (33) are computed after step 3c of Algorithm 3. The markers "circle" and "asterisk" in the figure for $\rho=5 w_{s}$ and $10 w_{s}$, respectively, represent the start of ADMM iteration for a new point $\left(\left\{\hat{\mathbf{z}}_{n l}\right\}_{n \in \mathcal{N}, l \in \mathcal{L}(n)},\left\{\hat{\mathbf{b}}_{n}\right\}_{n \in \mathcal{N}}\right)$ that is set at step 2 of the algorithm. Results show that the proposed distributed algorithm converges to the centralized objective value for different values of $\rho$. Furthermore, results show that a number of iterations between two successive "circle" markers (and also between two successive "asterisk" markers) reduces as the algorithm progress. That is, a fewer number of ADMM iterations (i.e., step 3 of Algorithm 3) is required as the algorithm progress.

In order to see the average behavior of Algorithm 2 and Algorithm 3, we next consider the fading case. For Algorithm 3, we set $\rho=w_{s}, 5 w_{s}$, and $10 w_{s}$. Let $\breve{f}_{0}^{k}$ denote the objective value of problem (46) obtained at $k$ th iteration, i.e., $\breve{f}_{0}^{k}=f_{0}\left(\left\{\mathbf{u}_{n l}^{k}, \mathbf{b}_{n}^{k}, \theta_{n}^{k}\right\}_{n \in \mathcal{N}, l \in \mathcal{L}(n)}\right)-$ $g_{0}\left(\left\{\mathbf{z}_{n l}^{k}, \mathbf{b}_{n}^{k}\right\}_{n \in \mathcal{N}, l \in \mathcal{L}(n)}\right)$; we stop both algorithms when either ${ }^{21}$ $\left|\breve{f}_{0}^{k+1}-\breve{f}_{0}^{k}\right| /\left|\breve{f}_{0}^{k}\right| \leq 0.001$, or algorithms run for a maximum of 25 iterations (see step 4 of both algorithms). To the best of our knowledge there is no algorithm for joint subchannel allocation and beamforming design for problem (33) in literature. Thus, as a benchmark, we consider the zero-forcing beamforming (ZFBF) with user selection algorithm proposed in [56, Sec. VI.B], and partition the $S$ subchannels among the operators by exhaustive search and random allocation. In random subchannel allocations, the subchannels are partitioned between the operators such that each of them obtain equal amount of subchannels. We refer to algorithm [56, Sec. VI.B] combined with an exhaustive search for partitioning subchannels as exhaustive-ZFBF; and the algorithm [56, Sec. VI.B] combined with a random channel allocation as random-ZFBF. We run all algorithms for 500 fading realizations.

\footnotetext{
${ }^{21} \mathrm{We}$ use relative stopping criteria because it is scaleindependent [Sec. 8.2], [55].
} 


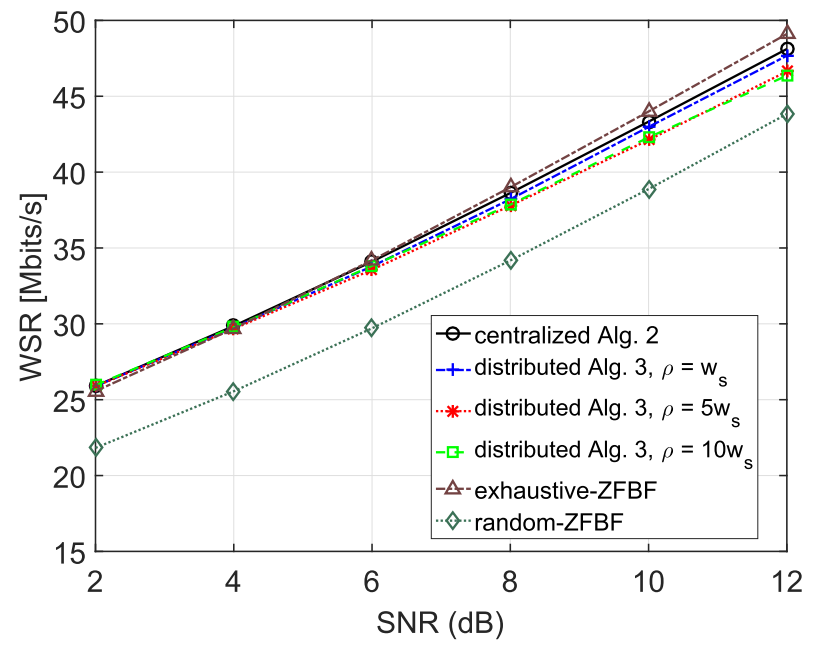

Fig. 5. Average WSR [Mbits/s] versus SNR.

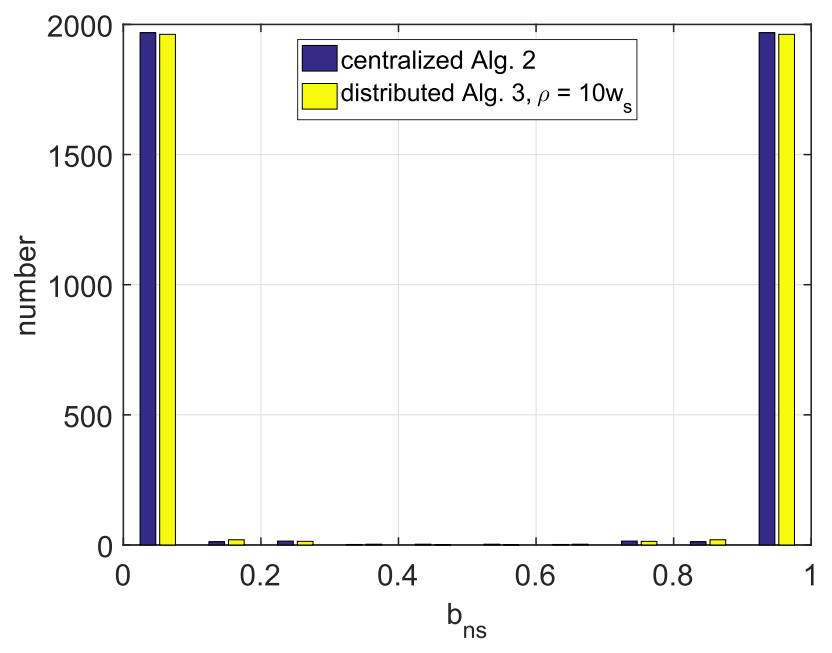

Fig. 6. Distribution of variables $\left\{b_{n s}\right\}_{n \in \mathcal{K}, s \in \mathcal{S}}$ for $\mathrm{SNR}=10 \mathrm{~dB}$.

Fig. 5 shows the average WSR, obtained by Algorithm 2 and Algorithm 3, versus SNR. Results show that the proposed algorithms perform slightly better than exhaustive-ZFBF at low to medium SNR values, while exhaustive-ZFBF performs better at high SNR values. However, exhaustive-ZFBF partitions the $S$ subchannels by exhaustive search, and its complexity is exponential in the number of subchannels. Thus, it quickly become intractable as the number of subchannels increases. On the other hand, both proposed algorithms outperform random-ZFBF for the entire range of SNR values. Furthermore, results show that distributed Algorithm 3 achieves WSR values closer to the centralized Algorithm 2.

Fig. 6 shows the distribution of variables $\left\{b_{n s}\right\}_{n \in \mathcal{N}, s \in \mathcal{S}}$. Results show that the values of $\left\{b_{n s}\right\}_{n \in \mathcal{N}, s \in \mathcal{S}}$ are (almost) either near to zero or one. Hence, the proposed algorithms can allocate $s$ th subchannel to a single operator. In the following simulations, if values of $\left\{b_{n s}\right\}_{n \in \mathcal{N}, s \in \mathcal{S}}$ are not exactly binary, we round it to the nearest binary value to assign $s$ th subchannel to a single operator.

We now evaluate the performance of Algorithm 1 . We suppose that the utility functions of users are given by

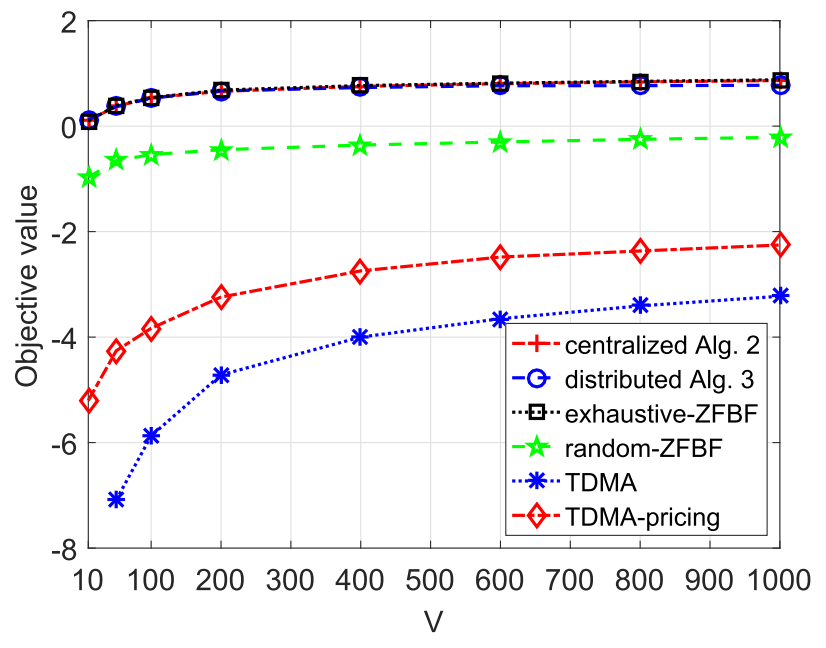

Fig. 7. Objective values of problem (7) versus parameter $V$.

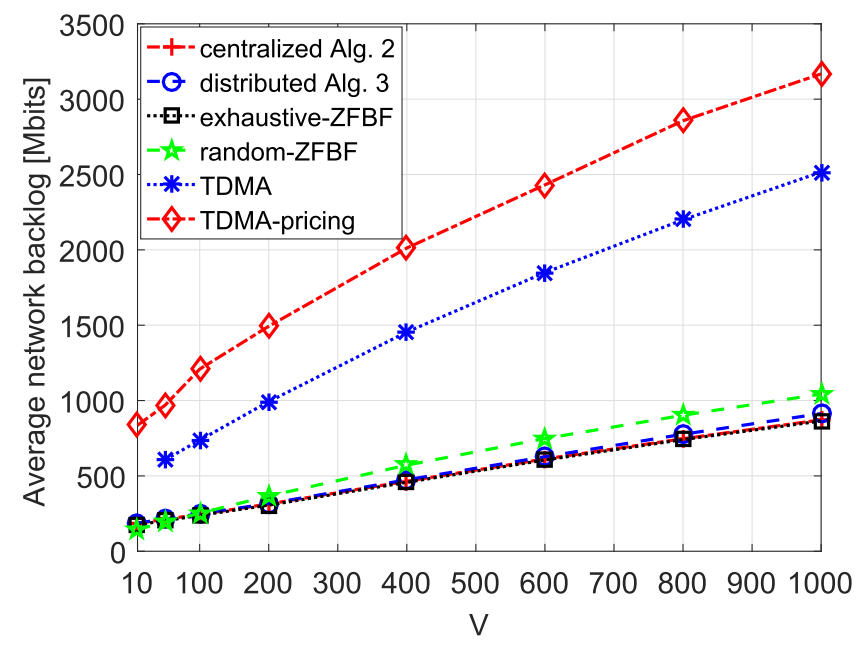

Fig. 8. Average network backlog versus parameter $V$.

$g_{n l}\left(a_{n l}\right)=\log \left(1+a_{n l}\right)$ for all $n \in \mathcal{N}$ and $l \in \mathcal{L}(n)$. The parameter $A^{\max }$ is computed as described in [23, Sec. 4.2.1], and it is given by $A^{\max }=S w_{s} \log _{2}\left(1+p_{0}^{\max } / N_{0} w_{s}\right)$. In fact, parameter $A^{\max }$ is an upper bound on the total transmission rate obtained by using $S$ subchannels with the transmit power $p^{\max }$. The maximum per-unit price of the spectrum band is set to $q^{\max }=g_{n l}\left(A^{\max }\right)$ [unit/MHz]. ${ }^{22}$ The parameter $\mu^{\max }$ is set such that it contains the optimal value of $\bar{U}_{n}-U_{n}^{0}$ for all $n \in \mathcal{N}\left[29\right.$, Ch. 5]. Since $A^{\mathrm{max}}$ is an upper bound on the total transmission rate, we have $\bar{U}_{n}-U_{n}^{0} \leq A^{\max }+q^{\max } B .{ }^{23}$ Thus, we set $\mu^{\max }=A^{\max }+q^{\max } B$. A value of disagreement point $U_{n}^{0}$ is obtained by solving a problem in Appendix. For simplicity, we assume that the transport layer storage reservoirs are saturated, i.e., there is always enough data waiting to be sent.

We run centralized and distributed versions of Algorithm 1 for $T^{\max }=1000$ time slots (fading realizations). Centralized

\footnotetext{
${ }^{22}$ The proposed dynamic algorithms are independent of the unit of price, and hence we work with a normalized unit. But the results could be scaled with a proper value of price-unit that operators mutually agree.

${ }^{23}$ Under network stability $\sum_{l \in \mathcal{L}(n)} \bar{a}_{n l}(t) \leq A^{\mathrm{max}}$ for all $n \in \mathcal{N}$. Hence from expression (6), we have $\bar{U}_{n} \leq A^{\max }+q^{\max } B$ for all $n \in \mathcal{N}$.
} 
version of Algorithm 1 is obtained by solving step 4 of the algorithm by using Algorithm 2. Distributed version of Algorithm 1 is obtained by solving step 4 of the algorithm by using Algorithm 3. As a benchmark, we consider the exhaustive-ZFBF and random-ZFBF algorithms to solve step 4 of Algorithm 1. Furthermore, to evaluate the performance of the proposed algorithms, we also consider timedivision multiple access (TDMA) approach to solve step 4 of Algorithm 1; specifically, operators are allowed to fully access the common spectrum pool in alternating time slots. We run Algorithm 1 embedded with TDMA approach, with and without considering the spectrum pricing strategy of Section II-A, for $T^{\max }=4000$ time slots. ${ }^{24}$ In the simulations, we set $\mathrm{SNR}=10 \mathrm{~dB}$, and the ADMM penalty parameter $\rho=10 w_{s}$.

Fig. 7 shows the objective values of problem (7) versus parameter $V$. Results show that the objective value improves as $V$ increases. Fig. 8 shows the time average network back$\log$, i.e., $1 / T^{\max } \sum_{\tau=1}^{T^{\max }} \sum_{n \in \mathcal{N}} \sum_{l \in \mathcal{L}(n)} Q_{n l}(\tau)$ versus parameter $V$. Results show that the time average network backlog increases with the parameter $V$. For TDMA based algorithm, the plots are drawn starting from $V=50$, as below this value the algorithm is unable to obtain operators' utilities above the disagreement points. ${ }^{25}$ Fig. 7 and Fig. 8 show that there is a trade-off between the achieved objective value of problem (7) and the network congestion. That is when the objective value increases, the network backlog is also increased. Furthermore, results show that both centralized and distributed versions of Algorithm 1 perform almost the same, and very close to that obtained by using exhaustive$Z F B F$ based dynamic control algorithm. However, proposed centralized and distributed versions of Algorithm 1 outperform control algorithms that are implemented using random-ZFBF, TDMA, and TDMA with pricing (TDMA-pricing) to solve step 4 of Algorithm 1. In addition, the TDMA based algorithms show that the pricing strategy of Section II-A improves the objective of problem (7) while increasing the queue backlog.

Fig. 9 shows the average profits of the operators $\left\{\bar{U}_{n}\right\}_{n \in \mathcal{N}}$ versus parameter $V$. Results show that the average profits of both the operators obtained by sharing their spectrum band with each other are greater than their disagreement points, i.e., $\bar{U}_{n} \geq U_{n}^{0}$ for all $n \in \mathcal{N}$. In other words, both operators gain in their profits by sharing their licensed spectrum band with each other, rather than using them exclusively. Furthermore, results show that the proposed algorithms outperform the control algorithms that are implemented by solving step 4 of Algorithm 1 with TDMA approach. Moreover, results show that when Algorithm 1 is embedded with TDMA approach, the operator with low spectrum demand (see operator 1) increases its profit by using the pricing strategy of Section II-A. That is the operator with low spectrum demand is getting paid for leasing its spectrum. Figure shows that the profit of each operator converges to its maximum value as $V$ increases.

\footnotetext{
${ }^{24}$ We used larger averaging window as TDMA based algorithm requires slightly longer time to reach a near steady state [29].

${ }^{25}$ Recall that in Algorithm 1 the value of $V$ puts emphasis on the objective of problem (7), see expression (19).
}

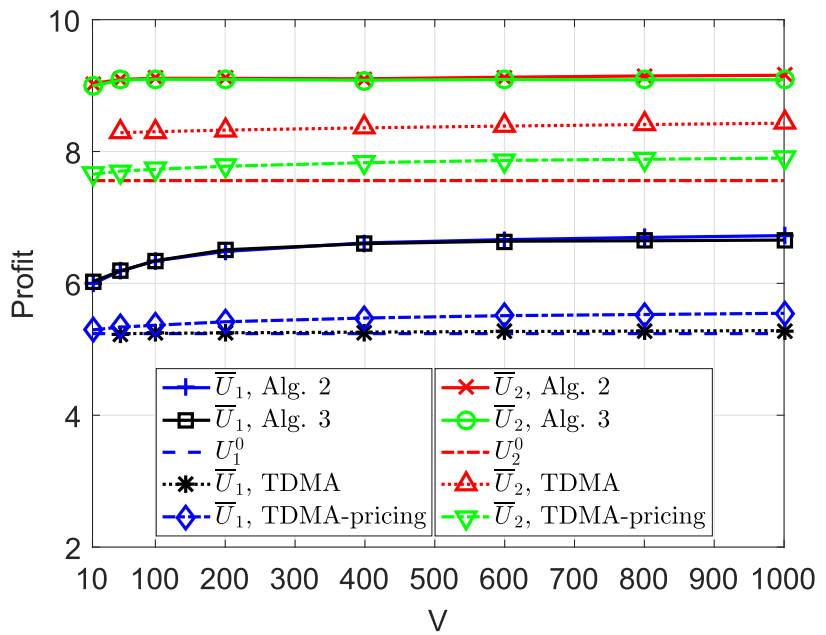

Fig. 9. Profits of the operators versus parameter $V$.

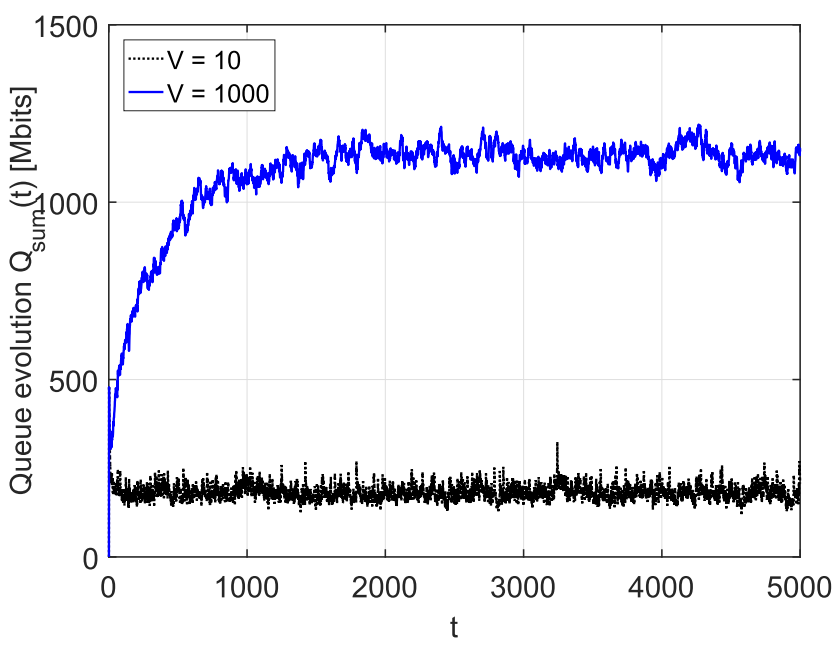

Fig. 10. Queue evolution $Q_{\text {sum }}(t)$ for $V=10$ and 1000 .

Fig. 10 shows the evolution of the network queue back$\log$ defined as $Q_{\text {sum }}(t)=\sum_{n \in \mathcal{N}} \sum_{l \in L(n)} Q_{n l}(t)$. Here, step 4 of Algorithm 1 is solved by using Algorithm 2. The algorithm is run for $V=10$ and 1000 . Results show that the network queue backlog $Q_{\text {sum }}(t)$ increases until it reaches a certain value (e.g., for $V=1000$ around 1400 Mbits), and then it oscillates. This is because of the negative drift property of the Lyapunov function [23, Ch. 4.4], and it ensures all queues are bounded and the network is stable.

\section{CONCLUSION}

We have considered a spectrum sharing problem between two operators in a dynamic network environment. We have allowed both operators to share their licensed spectrum bands with each other by forming a common spectrum band. Two-person bargaining framework has been used to model the spectrum sharing problem, and we have cast it as a stochastic optimization problem. To solve this problem, we have proposed both centralized and distributed dynamic control algorithms by using Lyapunov optimization. Numerically, we have shown that the proposed distributed algorithm achieves almost 
the same performance as the centralized one. Furthermore, it has been shown that operators gain in their profits by sharing their licensed spectrum band with each other, rather than using them exclusively; and there is a trade-off between the achieved profits of the operators and the network congestion. The proposed algorithms can be extended for multiple operators by redefining appropriately the spectrum pricing strategy of Section II-A. However, this extension is nontrivial and it falls outside the main scope of this paper; thus it is left as a future interesting work.

\section{APPENDIX}

We set $U_{n}^{0}$ to a value of utility that $n$th operator gain by using $B \mathrm{~Hz}$ of spectrum band (i.e., without sharing its spectrum band with other operator). The resource allocation problem for $n$th operator without sharing its licensed spectrum band can be obtained by modifying problem (32). Specifically, by dropping the constraint associated with orthogonal subchannel allocation of problem (32), and the payment terms with the spectrum pricing in the objective function, the resource allocation problem for operator $n \in \mathcal{N}$ during time slot $t$ can be expressed as

$$
\begin{aligned}
\operatorname{maximize} & \sum_{l \in \mathcal{L}(n)} Q_{n l}(t) \sum_{s \in \mathcal{S}(n, t)} w_{s} \log _{2}(1 \\
& \left.+\frac{\left|\mathbf{h}_{n l, s}^{\mathrm{H}}(t) \mathbf{m}_{n l, s}\right|^{2}}{N_{0} w_{s}+\sum_{j \in \mathcal{L}(n), j \neq l}\left|\mathbf{h}_{n l, s}^{\mathrm{H}}(t) \mathbf{m}_{n j, s}\right|^{2}}\right)
\end{aligned}
$$$$
\text { subject to } \sum_{l \in \mathcal{L}(n)} \sum_{s \in \mathcal{S}(n, t)}\left\|\mathbf{m}_{n l, s}\right\|_{2}^{2} \leq p_{n}^{\max } \text {, }
$$

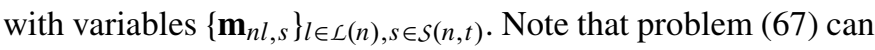
be solved with the approach presented in Section IV. Let us denote $\left\{\mathbf{m}_{n l, s}^{\star}(t)\right\}_{l \in \mathcal{L}(n), s \in \mathcal{S}(n, t)}$ the solution of problem (67), and the transmission rate be $r_{n l}^{\star}(t)$ of $l$ th user of $n$th operator (transmission rate can be calculated by using expression (2)). Let $\bar{r}_{n l}(t)$ denotes the time average rate defined as $\bar{r}_{n l}(t)=$ $\frac{1}{t} \sum_{\tau=1}^{t} r_{n l}^{\star}(\tau)$. Then the utility gain of $l$ th user of $n$th operator based on its current data rate $\bar{r}_{n l}(t)$ is $g_{n l}\left(\bar{r}_{n l}(t)\right)$. To estimate a value of $\left\{U_{n}^{0}\right\}_{n \in \mathcal{N}}$, we solve problem (67) with $Q_{n l}(t)=1$ for all $n \in \mathcal{N}$ and $l \in \mathcal{L}(n)$, and run simulation for 5000 fading realizations. Then a disagreement point for $n$th operator is set to $U_{n}^{0}=\sum_{l \in \mathcal{L}(n)} g_{n l}\left(\bar{r}_{n l}(5000)\right)$.

\section{REFERENCES}

[1] Notice for Proposed Rulemaking and Order: In the Matter of Facilitating Opportunities for Flexible, Efficient, and Reliable Spectrum Use Employing Cognitive Radio Technologies, Federal Commun. Commission, Washington, DC, USA, Tech. Rep. ET Docket No. 03-108, Dec. 2003.

[2] I. F. Akyildiz, W.-Y. Lee, M. C. Vuran, and S. Mohanty, "A survey on spectrum management in cognitive radio networks," IEEE Commun. Mag., vol. 46, no. 4, pp. 40-48, Apr. 2008.

[3] Intermediate Description of the Spectrum Needs and Usage Principles, document ICT-317669-METIS/D5.1, METIS, 2013.

[4] E. A. Jorswieck and et al., "Resource sharing in wireless networks: The SAPHYRE approach," in Proc. Future Netw. Mobile Summit, Jun. 2010, pp. $1-8$.

[5] E. A. Jorswieck, L. Badia, T. Fahldieck, E. Karipidis, and J. Luo, "Spectrum sharing improves the network efficiency for cellular operators," IEEE Commun. Mag., vol. 52, no. 3, pp. 129-136, Mar. 2014.

[6] J. Lindblom and E. G. Larsson, "Does non-orthogonal spectrum sharing in the same cell improve the sum-rate of wireless operators?" in Proc. IEEE Workshop Signal Adv. Wireless Commun., Jun. 2012, pp. 6-10.
[7] R. Gangula, D. Gesbert, J. Lindblom, and E. G. Larsson, "On the value of spectrum sharing among operators in multicell networks," in Proc. IEEE Veh. Technol. Conf., Jun. 2013, pp. 1-5.

[8] G. Middleton, K. Hooli, A. Tolli, and J. Lilleberg, "Inter-operator spectrum sharing in a broadband cellular network," in Proc. IEEE Int. Symp. Spread Spectr. Techn. Appl., Aug. 2006, pp. 376-380.

[9] M. Bennis and J. Lilleberg, "Inter base station resource sharing and improving the overall efficiency of B3G systems," in Proc. IEEE Veh. Technol. Conf., Sep./Oct. 2007, pp. 1494-1498.

[10] H. Kamal, M. Coupechoux, and P. Godlewski, "Inter-operator spectrum sharing for cellular networks using game theory," in Proc. IEEE Int. Symp. Pers., Indoor, Mobile Radio Commun., Sep. 2009, pp. $425-429$.

[11] L. Anchora, L. Badia, E. Karipidis, and M. Zorzi, "Capacity gains due to orthogonal spectrum sharing in multi-operator LTE cellular networks," in Proc. Int. Symp. Wireless Commun. Syst., Aug. 2012, pp. 286-290.

[12] J. E. Suris, L. A. DaSilva, Z. Han, and A. B. MacKenzie, "Cooperative game theory for distributed spectrum sharing," in Proc. IEEE Int. Conf. Commun., Jun. 2007, pp. 5282-5287.

[13] P. Luoto, P. Pirinen, M. Bennis, S. Samarakoon, S. Scott, and M. Latva-Aho, "Co-primary multi-operator resource sharing for small cell networks," IEEE Trans. Wireless Commun., vol. 14, no. 6, pp. 3120-3130, Jun. 2015.

[14] T. A. Weiss and F. K. Jondral, "Spectrum pooling: An innovative strategy for the enhancement of spectrum efficiency," IEEE Commun. Mag., vol. 42, no. 3, pp. S8-S14, Mar. 2004.

[15] P. Si, H. Ji, F. R. Yu, and V. C. M. Leung, "Optimal cooperative internetwork spectrum sharing for cognitive radio systems with spectrum pooling," IEEE Trans. Veh. Technol., vol. 59, no. 4, pp. 1760-1768, May 2010.

[16] M. J. Osborne and A. Rubinstein, A Course in Game Theory. Cambridge, MA, USA: MIT Press, 1994.

[17] Z. Han, Z. Ji, and K. J. R. Liu, "Fair multiuser channel allocation for OFDMA networks using Nash bargaining solutions and coalitions," IEEE Trans. Commun., vol. 53, no. 8, pp. 1366-1376, Aug. 2005.

[18] A. Leshem and E. Zehavi, "Bargaining over the interference channel," in Proc. IEEE Int. Symp. Inf. Theory, Jul. 2006, pp. 2225-2229.

[19] Z. Ka, M. Ho, and D. Gesbert, "Spectrum sharing in multiple-antenna channels: A distributed cooperative game theoretic approach," in Proc. IEEE Int. Symp. Pers., Indoor, Mobile Radio Commun., Sep. 2008, pp. 1-5.

[20] E. G. Larsson and E. A. Jorswieck, "Competition versus cooperation on the MISO interference channel," IEEE J. Sel. Areas Commun., vol. 26 no. 7, pp. 1059-1069, Sep. 2008.

[21] J. Luo and et al., "Transmit beamforming for inter-operator spectrum sharing: From theory to practice," in Proc. Int. Symp. Wireless Commun. Syst., Aug. 2012, pp. 291-295.

[22] E. Karipidis et al., "Transmit beamforming for inter-operator spectrum sharing," in Proc. Future Netw. Mobile Summit (FutureNetw), Jun. 2011, pp. 1-8.

[23] L. Georgiadis, M. J. Neely, and L. Tassiulas, "Resource allocation and cross-layer control in wireless networks," Found. Trends Netw., vol. 1, no. 1, pp. 1-144, Apr. 2006.

[24] Y. Teng, Y. Wang, and K. Horneman, "Co-primary spectrum sharing for denser networks in local area," in Proc. IEEE Int. Conf. Cognit. Radio Oriented Wireless Netw. Commun., Jun. 2014, pp. 120-124.

[25] J. F. Nash, Jr., "The bargaining problem," Econometrica, vol. 18, no. 2, pp. 155-162, 1950.

[26] L. Gao, G. Iosifidis, J. Huang, L. Tassiulas, and D. Li, "Bargainingbased mobile data offloading," IEEE J. Sel. Areas Commun., vol. 32, no. 6, pp. 1114-1125, Jun. 2014.

[27] H. Boche and M. Schubert, "A generalization of Nash bargaining and proportional fairness to log-convex utility sets with power constraints," IEEE Trans. Inf. Theory, vol. 57, no. 6, pp. 3390-3404, Jun. 2011.

[28] V. V. Vazirani, "The notion of a rational convex program, and an algorithm for the arrow-debreu Nash bargaining game," in Proc. ACM-SIAM Symp. Discrete Algorithms, 2012, pp. 973-992.

[29] M. J. Neely, Stochastic Network Optimization With Application to Communication and Queueing Systems (Synthesis Lectures on Communication Networks), vol. 7. San Rafael, CA, USA: Morgan \& Claypool, 2010.

[30] Z.-Q. Luo and S. Zhang, "Dynamic spectrum management: Complexity and duality," IEEE J. Sel. Topics Signal Process., vol. 2, no. 1, pp. 57-73, Feb. 2008. 
[31] S. Boyd. (2007). Sequential Convex Programming. [Online]. Available: http://www.stanford.edu/class/ee364b/lectures/seq_slides.pdf

[32] S. Boyd, N. Parikh, E. Chu, B. Peleato, and J. Eckstein, "Distributed optimization and statistical learning via the alternating direction method of multipliers," Found. Trends Mach. Learn., vol. 3, no. 1, pp. 1-122, Jan. 2011.

[33] Future Spectrum System Concept, document ICT-317669-METIS/D5.4, METIS, 2015.

[34] D. Tse and P. Viswanath, Fundamentals of Wireless Communication. Cambridge, U.K.: Cambridge Univ. Press, 2005.

[35] M. J. Neely, "Dynamic power allocation and routing for satellite and wireless networks with time varying channels," Ph.D. dissertation, Dept. Elect. Eng. Comput. Sci., Massachusetts Inst. Technol., Cambridge, MA, USA, 2003.

[36] M. J. Neely, "Super-fast delay tradeoffs for utility optimal fair scheduling in wireless networks," IEEE J. Sel. Areas Commun., vol. 24, no. 8, pp. 1489-1501, Aug. 2006.

[37] Y. Mao, J. Zhang, and K. B. Letaief, "A Lyapunov optimization approach for green cellular networks with hybrid energy supplies," IEEE J. Sel. Areas Commun., vol. 33, no. 12, pp. 2463-2477, Dec. 2015.

[38] T. Shuminoski and T. Janevski, "Lyapunov optimization framework for 5G mobile nodes with multi-homing," IEEE Commun. Lett., vol. 20, no. 5, pp. 1026-1029, May 2016.

[39] S. Li, J. Huang, and S.-Y. Li, "Dynamic profit maximization of cognitive mobile virtual network operator," IEEE Trans. Mobile Comput., vol. 13, no. 3, pp. 526-540, Mar. 2014.

[40] S. Lakshminarayana, T. Q. S. Quek, and H. V. Poor, "Cooperation and storage tradeoffs in power grids with renewable energy resources," IEEE J. Sel. Areas Commun., vol. 32, no. 7, pp. 1386-1397, Jul. 2014.

[41] J. Li, J. Wu, M. Peng, and P. Zhang, "Queue-aware energy-efficient joint remote radio head activation and beamforming in cloud radio access networks," IEEE Trans. Wireless Commun., vol. 15, no. 6 , pp. 3880-3894, Jun. 2016.

[42] F. P. Kelly, A. Maulloo, and D. Tan, "Rate control for communication networks: Shadow prices, proportional fairness, and stability," J. Oper. Res. Soc., vol. 49, no. 3, pp. 237-252, 1998.

[43] T. Bu, L. Li, and R. Ramjee, "Generalized proportional fair scheduling in third generation wireless data networks," in Proc. IEEE Int. Conf. Comput. Сотти., Apr. 2006, pp. 1-12.

[44] M. Neely, "Optimal pricing in a free market wireless network," in Proc. IEEE Int. Conf. Comput. Commun., May 2007, pp. 213-221.

[45] M. Peng, X. Xie, Q. Hu, J. Zhang, and H. V. Poor, "Contractbased interference coordination in heterogeneous cloud radio access networks," IEEE J. Sel. Areas Commun., vol. 33, no. 6, pp. 1140-1153, Jun. 2015.

[46] T. Lipp and S. Boyd, "Variations and extension of the convex-concave procedure," J. Optim. Eng., vol. 17, no. 2, pp. 263-287, 2016.

[47] Z.-Q. Luo, W.-K. Ma, A. M.-C. So, Y. Ye, and S. Zhang, "Semidefinite relaxation of quadratic optimization problems," IEEE Signal Process. Mag., vol. 27, no. 3, pp. 20-34, May 2010.

[48] P. C. Weeraddana, M. Codreanu, M. Latva-Aho, and A. Ephremides, "Multicell MISO downlink weighted sum-rate maximization: A distributed approach," IEEE Trans. Signal Process., vol. 61, no. 3, pp. 556-570, Feb. 2013.

[49] N. D. Sidiropoulos, T. N. Davidson, and Z.-Q. Luo, "Transmit beamforming for physical-layer multicasting," IEEE Trans. Signal Process., vol. 54, no. 6, pp. 2239-2251, Jun. 2006.

[50] S. Boyd and L. Vandenberghe, Convex Optimization. Cambridge, U.K.: Cambridge Univ. Press, 2004.

[51] D. P. Bertsekas and J. N. Tsitsiklis, Parallel and Distributed Computation: Numerical Methods, 2nd ed. Belmont, MA, USA: Athena Scientific, 1997.

[52] Isolation Between Antennas of IMT Base Stations in the Land Mobile Service, document ITU-R M.2244, ITU, Geneva, Swizerland, 2011

[53] "Mobile infrastructure sharing," GSMA Foundation, London, U.K. Tech. Rep. [Online]. Available: https://www.gsma.com/publicpolicy/wpcontent/uploads/2012/09/Mobile-Infrastructure-sharing.pdf

[54] A. Kumar, D. Manjunath, and J. Kuri, Wireless Networking. Burlington, MA, USA: Elsevier, 2008.

[55] E. K. P. Chong and S. H. Zak, An Introduction to Optimization, 2nd ed. New York, NY, USA: Wiley, 2001.

[56] T. Yoo and A. Goldsmith, "On the optimality of multiantenna broadcast scheduling using zero-forcing beamforming," IEEE J. Sel. Areas Commun., vol. 24, no. 3, pp. 528-541, Mar. 2006. 